\title{
EL FUTURO ENERGÉTICO DE JAPÓN: ENTRE EL REGRESO A LA SENDA NUCLEAR Y EL GIRO HACIA LAS RENOVABLES
}

\author{
María Francisca Casado ${ }^{1}$ \\ Universidad Europea de Madrid
}

\begin{abstract}
Resumen:
Tras el triple desastre del 11 de marzo de 2011, Japón se encuentra en una encrucijada energética. A corto y medio plazo depende de las importaciones de combustibles fósiles para garantizar su seguridad energética, pero el largo plazo está por decidir y lo determinarán las decisiones que tome en estos años. Como consta en su Estrategia de Seguridad Nacional, para Japón la seguridad energética es una cuestión de seguridad nacional. El artículo revisa la trayectoria nuclear nipona, estudia cuáles son los factores que configuran el mercado eléctrico japonés y analiza su actual situación energética. Por otra parte, también repasa los principios que han marcado su política energética, deteniéndose a evaluar los dos últimos Planes Estratégicos de Energía - uno anterior y otro posterior a Fukushima- antes de adentrarse en el debate sobre la composición óptima del futuro mix energético para cumplir con su trilema de la seguridad energética, marcado por su compromiso con el medio ambiente.
\end{abstract}

Palabras clave: Seguridad energética, mix energético, Estrategia de Seguridad Nacional de Japón, Plan Estratégico de Energía, energía nuclear, energías renovables, Japón, Fukushima.

Tittle in English: "Japan's energy future: Between a return to nuclear energy and a turn to renewables"

\begin{abstract}
:
After the triple disaster of 11 March 2011, Japan is at an energy crossroad. In the short and medium run it depends on fossil fuel imports to ensure its energy security, but the long term will be determined by the decisions taken at present. For Japan energy security is a national security challenge, as stated in its National Security Strategy. The article reviews the Japanese nuclear path, studies the factors shaping the Japanese electricity market and analyzes the current energy situation. Moreover, it also assesses the principles that have marked Japan's energy policy and the two last Strategic Energy Plans -one prior to Fukushima and the other after it-before tackling the debate on the optimal future energy mix that Japan should adopt to meet its energy security trilemma, marked by its environmental commitment.
\end{abstract}

Keywords: Energy security, energy mix, National Security Strategy of Japan, Strategic Energy Plan, nuclear energy, renewable energies, Japan, Fukushima.

Copyright (C) UNISCI, 2016.

Las opiniones expresadas en estos artículos son propias de sus autores, y no reflejan necesariamente la opinión de UNISCI. The views expressed in these articles are those of the authors, and do not necessarily reflect the views of UNISCI.

\footnotetext{
${ }^{1}$ María Francisca Casado es Doctora en Paz y Seguridad Internacional por la UNED. Enseña en la Facultad de Ciencias Sociales de la Universidad Europea de Madrid.

E-mail: mariafrancisca.casado@gmail.com

DOI: http://dx.doi.org/10.5209/rev_RUNI.2016.n41.52675
} 


\section{Introducción}

Tras el terremoto y posterior tsunami del 11 de marzo de 2011 que provocaron el accidente nuclear de Fukushima, se procedió a una "parada en frío" ${ }^{2}$ temporal de todas las centrales nucleares y cambió de signo el sentimiento popular hacia una energía que se había promovido como totalmente segura. El accidente había puesto al descubierto tanto "la fragilidad y contradicciones de la política energética de Japón", como la visión de posguerra equivocadamente optimista según la cual los avances tecnológicos solucionarían los problemas asociados al modelo de desarrollo económico acelerado de Japón ${ }^{3}$, entre ellos el de asegurar el suministro de energía.

Japón importa prácticamente la totalidad de los combustibles fósiles que consume. La escasez de minerales y energía de Japón ha influido decisivamente en su política económica durante la mayor parte de su historia contemporánea. Tras su derrota en la Segunda Guerra Mundial, el país quedó destruido y su maltrecha economía afectada por el coste de las reparaciones de guerra. El gobierno se embarcó en un ambicioso proyecto de ponerse a la par con occidente (catching up with the West), cuyo éxito dependía de su capacidad para garantizar un suministro de electricidad constante, 24 horas al día 7 días a la semana, a fin de mantener en funcionamiento la maquinaria industrial. Desde la década de 1950 hasta la crisis del petróleo de 1973, el vertiginoso desarrollo industrial que dio lugar al "milagro japonés", se llevó a cabo a base de combustibles fósiles importados, con una fuerte dependencia del petróleo de Oriente Medio.

Los precios del crudo, hasta entonces razonables, se cuadriplicaron a causa del embargo petrolífero árabe, que también puso en riesgo el suministro. Japón que crecía a un ritmo del $10 \%$ anual temió por las repercusiones que ello pudiese acarrear a su boyante economía y, consiguientemente, a su sociedad. Hallar una alternativa al petróleo se convirtió en una cuestión se seguridad nacional, que pasaba por lograr la autosuficiencia. Japón se debatía así entre el miedo a lo nuclear y el deseo de ser independiente de los proveedores externos.

La crisis del petróleo de 1973 supuso un punto de inflexión. Japón ya contaba con cinco reactores en funcionamiento y un incipiente sector nuclear, por lo que decidió apostar por la energía nuclear a fin de reducir su dependencia exterior de fuentes de energía. El país, que en 1945 había sufrido los efectos del uso bélico de dicha energía tras la caída de sendas bombas atómicas en Hiroshima y Nagasaki, había dado un giro radical hacia el uso pacífico de la misma en parte gracias a la exitosa campaña propagandística Atoms for Peace ("Átomos por la paz") del gobierno Eisenhower. Durante años, gran parte del suministro eléctrico del archipiélago japonés procedió de la energía nuclear y, junto con Francia y Alemania, Japón se convirtió en paradigma de su uso como una de sus principales fuentes de energía primaria.

\footnotetext{
2 "Cierre en frío" o "parada fría", cold shutdown, es el término técnico con el que se designa la detención de un reactor en condiciones de seguridad tras enfriar hasta la temperatura más baja posible los materiales radioactivos de su núcleo. Los expertos enfatizan que el cierre en frío solo es posible en reactores que estén funcionando en perfectas condiciones, no sería un término adecuado para describir el enfriamiento de los reactores accidentados en Fukushima I.

3 Matanle, Peter: "The Great East Japan Earthquake, tsunami, and nuclear meltdown: towards the (re)construction of a safe, sustainable, and compassionate society in Japan's shrinking regions", Local Environment, vol. 16, $\mathrm{n}^{\circ} 9$ (octubre 2011), p. 837.

${ }^{4}$ Se denomina "milagro japonés" al rápido crecimiento industrial que propició la incorporación de Japón a la OCDE a comienzos de la década de 1970. En un tiempo menor del esperado, Japón había pasado de ser una economía en desarrollo a formar parte del club de naciones desarrolladas y, asimismo, el que había sido un país beligerante se integraba en el concierto mundial abogando por la paz, la estabilidad y los valores democráticos.
} 
Sin embargo, tras el "triple desastre" (así denominado por la confluencia de terremoto, tsunami y accidente nuclear) y debido a la parada en frío de los reactores nucleares, el gobierno japonés ha tenido que recurrir a los combustibles fósiles para satisfacer su demanda energética a corto y medio plazo. Las importaciones de combustibles fósiles han situado a Japón en lo alto del ranking de países importadores, aumentando su dependencia externa que ha vuelto a situarse en los niveles de la década de 1970. Como puede apreciarse en el siguiente gráfico, antes de la crisis petrolífera de 1973 Japón importaba el 75,5\% del petróleo, el $16,9 \%$ del carbón y el 1,6\% del GNL que consumía lo cual sumado ascendía a 89,7\%. La suma de los porcentajes de carbón $(23,4 \%)$, petróleo $(44,3 \%)$ y GNL $(24,5 \%)$ consumidos en 2012 también ronda el $90 \%$, ascendiendo a 92,2\%, lo cual nos da una idea de su fuerte dependencia externa.

\section{Gráfico 1. Fuentes de energía primaria en 1973 y 2012}

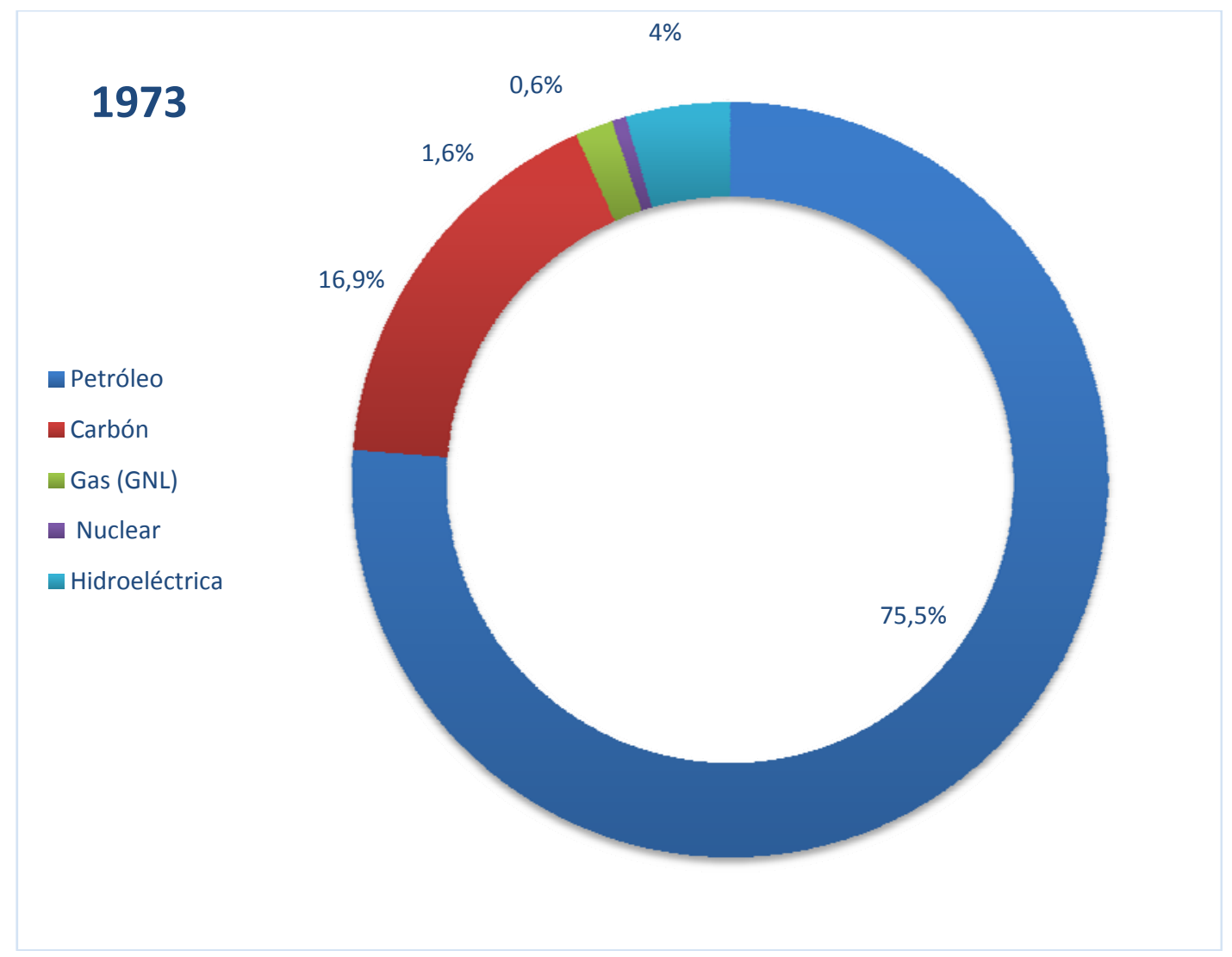

Fuente: METI 


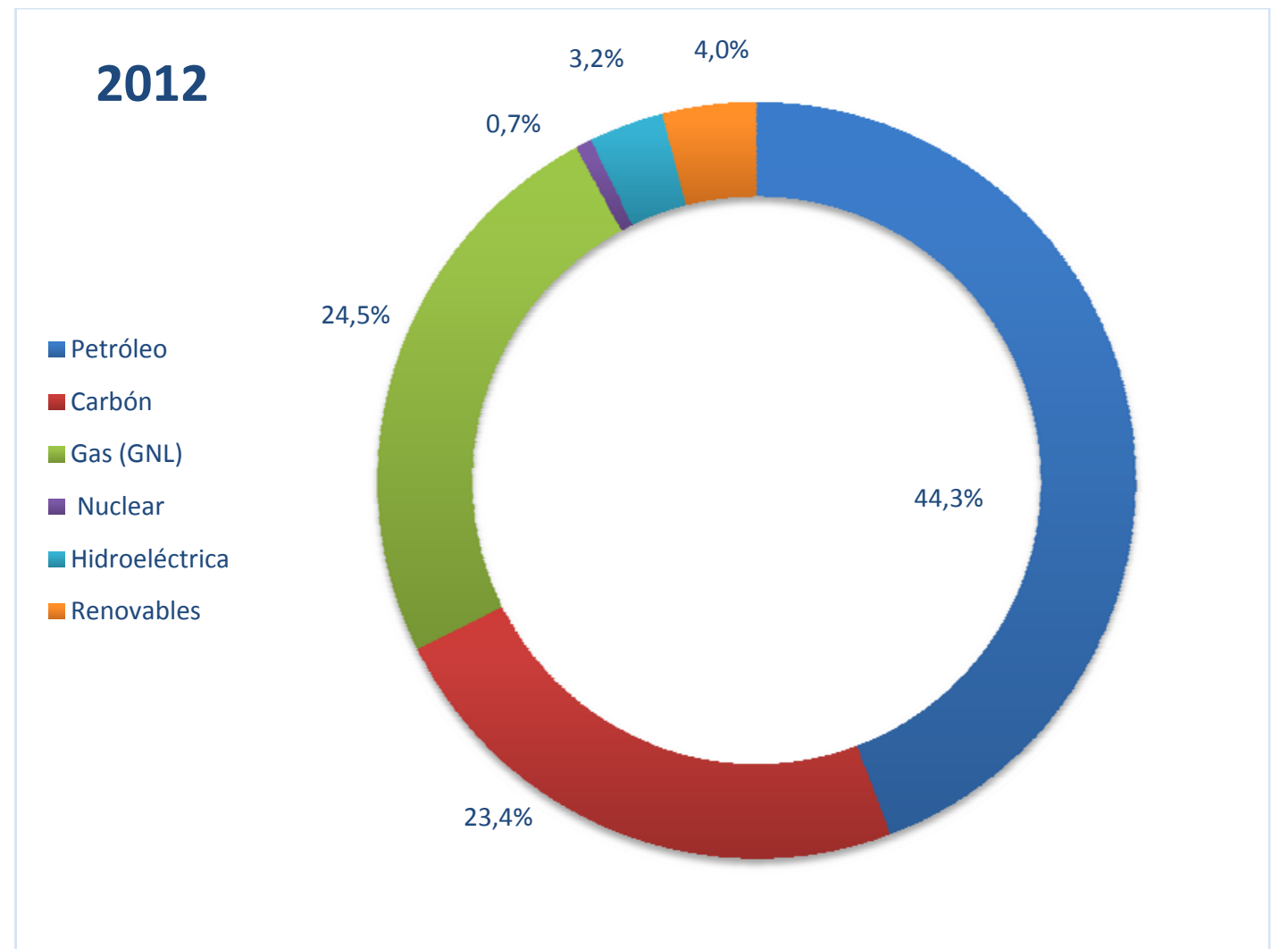

Fuente: METI

Para un país que importa el $90 \%$ de la energía que consume ${ }^{5}$, cuyo gobierno consideraba la energía nuclear una prioridad estratégica nacional desde 1973, asegurar un suministro eléctrico constante y asequible en condiciones de total seguridad, es un reto que en estos momentos está siendo tratado por las vías políticas ordinarias pero que también está presente en su estrategia nacional de seguridad.

Sin embargo, la demanda de energía ha ido en aumento en las últimas décadas a nivel mundial debido tanto al crecimiento de la población mundial, como al desarrollo económico que ha hecho que la demanda sea mayor en las nuevas economías industrializadas, en particular en los BRICs. Por tanto, en este contexto, cabe cuestionarse por un lado cómo va a hacer Japón frente a sus necesidades energéticas a largo plazo y, por el otro, cómo lo va a lograr en este escenario mundial.

\section{El trilema de la seguridad energética}

Ang, Choong y Ng han realizado una exhaustiva revisión de la literatura actual (hasta 2015) existente en inglés sobre seguridad energética, así como sobre los indicadores utilizados para medirla. Tras identificar 83 definiciones de seguridad energética, encontraron en su análisis tanto gran diversidad entre las mismas, como siete temas básicos entre los que destacaba el de la disponibilidad de energía, que se repetía en casi todas las definiciones. Además, apreciaron que el alcance de la seguridad energética se extendía a asuntos que en años anteriores no se tomaban en consideración, como el medio ambiente, la gobernabilidad y la eficiencia energética; siendo el tratamiento de los mismos "holístico" lo cual a su vez influía en la construcción de los indicadores que miden la seguridad energética. En este sentido, también identificaron en su revisión de la literatura 53 estudios que trataban el tema de los indicadores;

\footnotetext{
${ }^{5}$ Datos de JAIF (Japan Atomic Industrial Forum).
} 
mientras que en algunos de ellos tan sólo se utilizaban unos pocos indicadores en otros se empleaban más de 60 . No obstante, en dos tercios de los estudios como media no se recurría a más de 20 indicadores de seguridad energética ${ }^{6}$.

Si bien esta reciente revisión de la literatura pone de manifiesto la ausencia de consenso en torno a la definición de seguridad energética, hemos de decantarnos por alguna y, por ello, tomamos como punto de partida la definición institucional de la Agencia Internacional de la Energía. La seguridad energética ha sido definida por la IEA como la necesidad de asegurar un suministro de energía suficiente a precios razonables. Según dicha Agencia, la seguridad energética presenta múltiples aspectos, a corto plazo supone asegurar la capacidad del sistema energético para reaccionar rápidamente a cambios repentinos en el equilibrio entre oferta y demanda, mientras que a largo plazo implica realizar las inversiones necesarias para garantizar un suministro energético que se ajuste a la evolución económica, teniendo en cuenta las necesidades medioambientales ${ }^{7}$. En la misma línea se encuentra la definición de Vivoda que la considera "la disponibilidad de energía en todo momento en varias formas, en suficientes cantidades y a precios asequibles, sin que tenga un efecto irreversible o inaceptable sobre la economía y sobre el medio ambiente" ${ }^{\natural}$.

En este sentido, hay que tomar en consideración que cada tipo de energía deja su huella en el medio ambiente en forma de residuos y gases de efecto invernadero y que, dado que la energía puede obtenerse de diferentes fuentes como son los combustibles fósiles, la energía hidroeléctrica, la energía nuclear o las energías renovables (solar, eólica, biomasa, geotérmica), quienes toman decisiones en materia de política energética deben encontrar una combinación equilibrada de fuentes energéticas o "mix de fuentes de energía" (en adelante "mix energético") que facilite el logro de objetivos tanto económicos como medioambientales", asegurando asimismo el suministro energético. A esta trilogía de objetivos, Ang et alia la denominan "Trilema de la Seguridad Energética" y lo representan de la siguiente manera:

\footnotetext{
${ }^{6}$ Ang, B. W., Choong, W. L. y Ng, T. S.: "Energy security: Definitions, dimensions and indexes", Renewable and Sustainable Energy Reviews, no 42 (febrero 2015), pp. 1077-1093, p. 1090.

${ }^{7}$ International Energy Agengy (IAE) en http://www.iea.org/topics/energysecurity/

${ }^{8}$ Vivoda, Vlado: “Japan's energy security predicament post-Fukushima”, Energy Policy, $n^{\circ} 46$ (julio 2012 ), p. 135.

9 A esta dualidad de objetivos se les denomina en inglés "Growth Environment dilemma" o "GE dilemma", el encontrar un equilibrio entre medio ambiente y crecimiento (económico) teniendo en cuenta las repercusiones negativas que el crecimiento tiene para el medio ambiente.
} 


\section{Gráfico 2. Trilema de la seguridad energética}

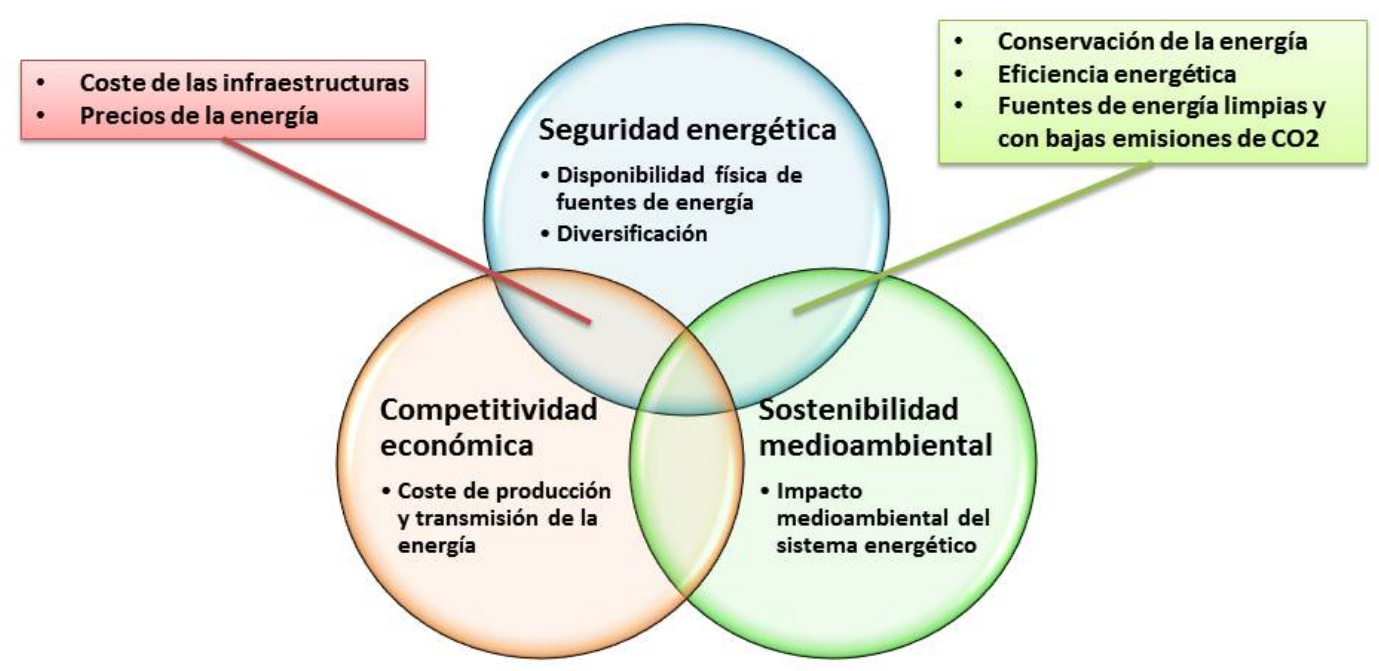

Fuente: Ang et al., op. cit., p. 1090

Durante años la política energética de Japón se ha basado en estos tres pilares ${ }^{10}$ conocidos como las "3 Es", en referencia a la iniciales en inglés de "seguridad energética" (Energy security), "medio ambiente" (Environment) y "eficiencia económica" (Economic efficiency), tendiendo a buscar el equilibro entre esos tres pilares ${ }^{11}$.

Tras el accidente de Fukushima, otros dos factores básicos han entrado en la ecuación: garantizar la seguridad (Safety) y el impacto macroeconómico. El primero se refiere ante todo a la seguridad de las centrales nucleares, que detuvieron su funcionamiento tras el desastre de Fukushima y antes de reiniciarse deberán pasar un exhaustivo examen, cumpliendo con requisitos más estrictos establecidos por la nueva Nuclear Regulation Authority (NRA), substituta de la Nuclear and Industrial Security Agency (NISA) ${ }^{12}$. Por su parte, el impacto

\footnotetext{
${ }^{10}$ Las “3 Es” ya estaban presentes en el la Ley Básica de Energía de 2002 y en el Primer Plan Estratégico de Energía aprobado en 2003, como veremos más adelante. Por otro lado, son esos tres pilares los que también conforman el trilema (seguridad, competitividad, sostenibilidad) de la seguridad energética de la Unión Europea (véase Herrero, Rubén: "Las cuestiones energéticas en la Estrategia de Seguridad Nacional 2013", UNISCI Discussion Papers, n⿳35 (mayo 2014), p. 370. y los mismos dentro de los cuales España de nuevo enmarca su Estrategia de Seguridad Energética Nacional 2015, aprobada en julio de 2015 (Estrategia de Seguridad Energética Nacional 2015, en http://www.lamoncloa.gob.es/serviciosdeprensa/notasprensa/Documents/ESTRATEGIA\%20DE\%20SEGURID AD\%20ENERG\%C3\%89TICA\%20NACIONAL\%20\%28WEB\%29.pdf).

${ }^{11}$ También la política energética de la Unión Europea y, por extensión, el gobierno español se han eco de este "trilema", alineando sus esfuerzos en este sentido. Véase Herrero, op. cit., pp. 367-379.

${ }^{12}$ En el momento del accidente de Fukushima, la antecesora de la NISA era un ente que dependía del METI, que a su vez era el encargado de promover la construcción de centrales nucleares. En otras palabas, desarrollo y evaluación recaían sobre el mismo ente, dando lugar a un conflicto de intereses más grave aún si tenemos en cuenta que en ocasiones los expertos encargados de hacer las evaluaciones eran los propios evaluados, pues la NISA carecía de expertos y recurría a las empresas eléctricas cuando necesitaba de conocimiento experto, un fenómeno que se conoce como "cautividad regulatoria". Una de las decisiones del Gabinete Kan fue la de
} 
macroeconómico está intrínsecamente ligado con el "mix energético" y con hallar una combinación del mismo que modere tanto su elevado coste, como su consiguiente efecto adverso sobre la recuperación económica del país, sin dejar de lado las preocupaciones medioambientales.

No obstante, la incorporación de estos principios a la política energética japonesa se ha llevado a cabo de forma gradual en el curso de los últimos cuarenta años y ha venido marcada por la necesidad de adaptarse al contexto en cada momento. Hagamos un breve repaso de este recorrido para ver como en cada momento la situación justificaba la adopción de estos principios:

- La década de 1970 se caracterizó por dos graves crisis energéticas, las de 1973 y 1979, motivadas por el ascenso de los precios del petróleo. Dada su escasez crónica de recursos y su fuerte dependencia externa, Japón puso el acento sobre la seguridad energética que siguió centrando su preocupación en la década siguiente.

- En la década de 1990 entró en juego la eficiencia económica, ya no sólo bastaba con garantizar el acceso a los preciados recursos energéticos, también había que asegurar su asequibilidad a fin de no lastrar ni el crecimiento del sector industrial, ni a las economías domésticas.

- En 1997 se adoptó el Protocolo de Kioto, que influyó también sobre la política energética japonesa; el dilema de la seguridad energética se transformó en un "trilema" al introducirse un nuevo elemento en la ecuación: el medio ambiente.

- A comienzos del siglo XXI, el trilema está más vigente que nunca, el gobierno pretende potenciar la seguridad de recursos para lo cual ha recurrido a la diversificación de proveedores, entre otros.

- En 2002, formalizando lo que ya se venía haciendo, el gobierno formula una Ley Básica de Energía, en la que se enmarcarán el primer plan energético y tres grevisiones del mismo, la última de marzo de 2014.

\section{La seguridad energética dentro de la Estrategia Nacional de Seguridad de Japón de 2013}

La última versión de la Estrategia Nacional de Seguridad de Japón ${ }^{13}$ de diciembre de 2013 sigue en la línea de anteriores documentos, revisando algunos planteamientos en relación a factores tanto internos como del entorno cercano y global. El documento comienza con una declaración de intenciones manifestando el interés del gobierno japonés por desempeñar un papel activo que contribuya a la paz basándose en los principios de cooperación internacional y alineando sus intereses a los de otras naciones democráticas, entre las que destaca a Estados Unidos, con las que comparte valores como "la libertad, la democracia, el respeto a los derechos humanos básicos y el Estado de Derecho".

Entre sus intereses nacionales, en línea con su declarado pacifismo, destaca el de alcanzar la prosperidad de Japón y de los japoneses a través del desarrollo económico, que a su vez propicie la consolidación de la paz y la seguridad. Para ello, es esencial que el entorno internacional ofrezca "estabilidad, transparencia y predictibilidad". El documento establece, asimismo, una serie de objetivos conducentes a lograr dichos intereses nacionales, estos

separar las tareas, de forma que la NRA se ha convertido en un organismo independiente, similar a los que operan en Estados Unidos y Francia, cuyas competencias ahora recaen en el Ministerio de Medio Ambiente. Los contenidos en inglés de su página web se pueden consultar en https:/www.nsr.go.jp/english/

${ }^{13}$ National Security Strategy of Japan (17 de diciembre de 2013), en: http://japan.kantei.go.jp/96 abe/documents/2013/ icsFiles/afieldfile/2013/12/17/NSS.pdf 
redactados en términos de seguridad tradicional o seguridad dura, utilizando términos como "disuasión" (deterrence), evitar que las "amenazas lleguen directamente a Japón" (deterring threats from directly reaching Japan) y "reducir amenazas directas a Japón fortaleciendo la Alianza Japón-Estados Unidos".

En su análisis del entorno de seguridad global y los retos que este representa, la Estrategia Nacional de Seguridad de Japón tiene en cuenta el cambio en el equilibrio de poder que supone el ascenso de China y el rápido progreso de las innovaciones tecnológicas, que otorgan mayor influencia a actores no estatales, como grandes corporaciones transnacionales $\mathrm{u}$ organizaciones criminales. Pero también menciona el riesgo en que se encuentran los "global commons" o bienes comunes globales sometidos a una mayor presión, esbozando la posibilidad de conflictos de intereses que podrían llevar a enfrentamientos por disputas territoriales o por el control de recursos. Otro riesgo que también tiene en cuenta el documento es el representa para la economía global el contagio de la crisis económica, con el consiguiente riesgo de que resurja el nacionalismo o de que se intensifique la competencia por los recursos minerales y energéticos.

Aunque la estabilidad del entorno y la economía parezcan ser las principales preocupaciones que refleja el documento, el gobierno japonés no pierde de vista el papel crucial que desempeña en ambas otra cuestión de fondo: la seguridad energética. Por una parte, en el plano político-económico, el ascenso de China conlleva un consumo de materias primas y energía para el que China deberá acudir a los mercados mundiales en competencia con otros países, incluido Japón. Asegurar su suministro también podría llevar a enfrentamientos como el que se está dando desde comienzos de 2015 en el Mar del Sur de China por la construcción de islas artificiales por parte de China en una zona cuya soberanía se disputan varias naciones, o el de las islas Senkaku/Diaoyou (entre China y Japón) en cuyo subsuelo se supone que podría haber grandes reservas minerales. Por tanto, implícitamente la seguridad energética está entre los temas que abarca el documento, donde también se trata explícitamente.

Concretamente en el Capítulo 5 que trata sobre el fortalecimiento de la cooperación basada en valores universales a fin de resolver cuestiones globales, en su quinto punto trata sobre la respuesta a cuestiones medioambientales y energéticas. Comienza reconociendo que, la necesidad para la dinámica economía japonesa de contar con un suministro estable de energía y otros recursos, "constituye un desafío para la seguridad nacional". Japón se planea tratar este asunto por medios diplomáticos, utilizando herramientas que permitan un acercamiento a los países productores de petróleo, carbón y gas natural y buscando una diversificación fuentes de abastecimiento que garantice un suministro constante a precios bajos. En el apartado, no deja de mencionar las implicaciones que ello conlleva para el medio ambiente (otra de las "Es" del trilema energético), manifestando su intención de respaldar a los países en desarrollo y de implementar una estrategia para contrarrestar el cambio climático tanto por medios tecnológicos, como mediante el establecimiento de un marco internacional para la reducción de emisiones.

Aunque la seguridad energética, como tema de seguridad está presente en la Estrategia Nacional de Seguridad de 2013, tanto implícita como explícitamente, su tratamiento no es por los cauces extraordinarios de la política exterior y de seguridad, sino vía los cauces ordinarios de la política energética que tratamos a continuación. Si bien antes nos detendremos a examinar la organización del sector eléctrico japonés. 


\section{Organización del sector energético japonés}

A fin de garantizar la seguridad energética del archipiélago japonés, el gobierno japonés ha fomentado y dirigido la participación de empresas privadas en todos los ámbitos de la cadena de suministro energético ${ }^{14}$. Al igual que en otros sectores económicos, la cadena de suministro energético está globalizada y consta de los siguientes procesos:

- Extracción de minerales y producción de energía bruta, que componen el upstream

- Gestión de oleoductos y gaseoductos, mantenimiento y administración de las infraestructuras de almacenamiento y transporte (el midstream)

- Generación, distribución y venta de energía (el downstream). En el caso del petróleo, es aquí donde se realiza el refinamiento del crudo y su conversión en derivados (gasolina, gasoil, diésel y demás), en el del gas su procesamiento y purificación.

En vista de la falta de fuentes nacionales de hidrocarburos, las empresas del sector energético han buscado tener una participación activa en el upstream a través de proyectos de gas natural y petróleo en todo el mundo, proporcionando servicios especializados de ingeniería, construcción, financiación y dirección de proyectos. No en vano, Japón es uno de los mayores exportadores de tecnología en el sector energético y posee un sólido programa de investigación, desarrollo e innovación $(\mathrm{I}+\mathrm{D}+\mathrm{i})$ respaldado por el gobierno. El gobierno también proporciona financiación a las empresas japonesas que ponen en marcha proyectos de exploración y producción, concediendo préstamos a interés favorable a través del Japan Bank for International Cooperation (JBIC) a fin de que estos puedan hacer efectivas sus ofertas de cara a conseguir proyectos en países productores de hidrocarburos clave, de esta manera las empresas japonesas pueden adquirir derechos de participación en yacimientos de petróleo y gas. Esto por una parte garantiza su propia seguridad energética y, por la otra, contribuye a asegurar el suministro ${ }^{15}$.

Por otro lado, grandes empresas privadas dominan el downstream, todas ellas japonesas dado que históricamente ha habido restricciones legales que han impedido a las empresas extranjeras acceder al mercado. Debido a la presión internacional, el panorama ha cambiado recientemente y el gobierno ha flexibilizado la legislación a fin de fomentar la competencia en las actividades de refinamiento del petróleo, permitiendo así la entrada de grandes empresas multinacionales del sector como Chevron, BP, Shell y BHP Billiton, entre otras. Estas multinacionales además de proporcionar derivados del petróleo y servicios al mercado japonés, son también socios en joint ventures con empresas japonesas en el exterior.

En cuanto a la electricidad, una de las peculiaridades de la red eléctrica japonesa es que, aunque en todo el territorio la tensión es de 110 voltios, en realidad cuenta con dos redes eléctricas, ya que el país está dividido en dos áreas, cada una de las cuales posee una frecuencia diferente. En la zona oeste de Japón la producción de electricidad es a 60 hercios, mientras que en la zona este es a 50 hercios $^{16}$. Además, como se aprecia en la Ilustración 3 , el

\footnotetext{
${ }^{14}$ En Japón existe un intercambio entre el sector privado (esfera empresarial) y el público (funcionarios y políticos) al que Richard Samuels en su libro de 1987, The business of the Japanese state: Energy markets in comparative and historical perspective denomina "consentimiento recíproco", del que se sirve para explicar y analizar la relación existente entre el Estado y el sector energético. El consentimiento recíproco es un proceso interactivo de negociaciones entre el mercado y el Estado en el que el sector privado toma parte en el proceso político y se beneficia de los recursos estatales (subsidios y ayudas), mientras el Estado a su vez posee cierto grado de jurisdicción sobre los sectores económicos.

${ }^{15}$ U.S. Energy Information Administration. (2015). Japan. (Country report), en http://www.eia.gov/beta/international/?fips=javolume-136-i-1832-english.pdf.

${ }^{16}$ En la zona oeste se encuentra la metrópoli de Tokio y la ciudad costera de Yokohama, mientras que en la zona oeste se hallan Osaka, Kioto y Kobe. En la actualidad Tokio es la capital económica y política de Japón y posee
} 
territorio japonés se reparte entre diez grandes operadoras eléctricas que tienen un control monopolístico sobre la producción, transmisión y comercialización de la electricidad. La mayor de ellas es TEPCO (Tokyo Electric Power Company por sus siglas en inglés), que es la empresa que suministra electricidad a Tokio y su área metropolitana, además de propietaria de la Central Nuclear de Fukushima.

\section{Gráfico 3. Red eléctrica de Japón.}

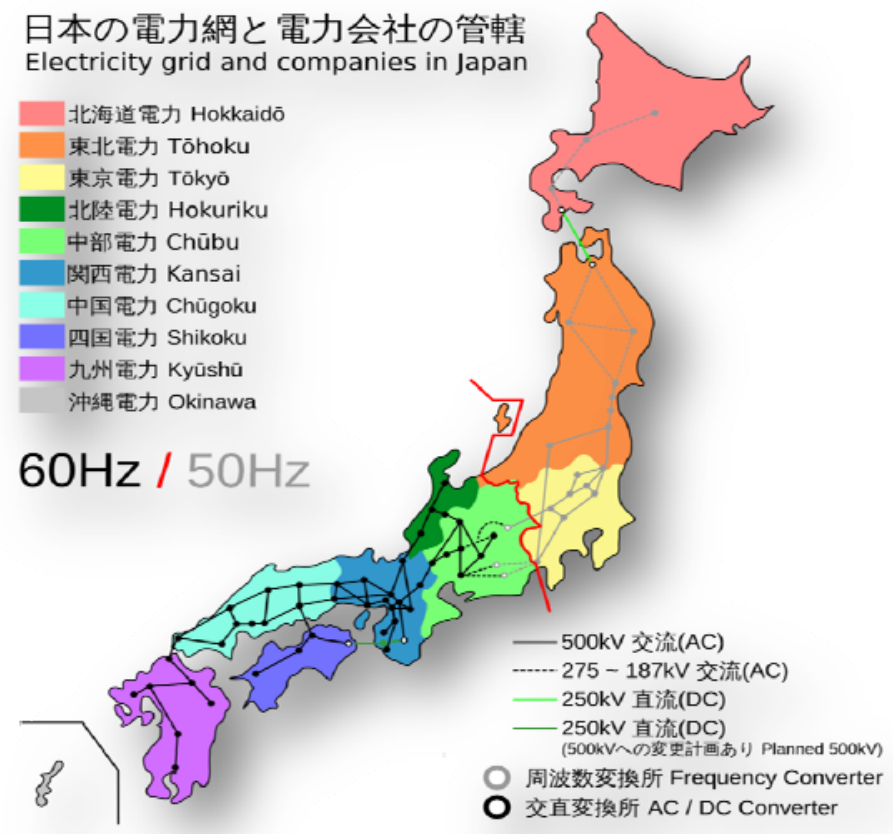

Fuente: Wikimedia, https://commons.wikimedia.org/wiki/File:Power_Grid_of_Japan.svg

Estas operadoras han seguido una estrategia de autosuficiencia orientada a maximizar sus propias instalaciones sin invertir en desarrollar la capacidad de transmisión inter-regional, por lo que existen pocos conversores y la conversión resulta demasiado cara. La dificultad para transmitir electricidad de un lado al otro del país, que se puso en evidencia a raíz del accidente nuclear de Fukushima, ha contribuido a que se agilicen los trámites para reestructurar el sector, algo que ya estaba en la agenda política antes del accidente. El accidente también dejó al descubierto otros aspectos negativos del sistema regional de monopolios como son la falta de competencia y el fuerte control sobre el precio, así como las limitaciones de las empresas eléctricas para poder efectuar un cambio en el mix energético a fin de potenciar el papel de las renovables.

\section{La política energética japonesa desde una perspectiva histórica}

Scalise califica la política energética japonesa y su regulación del sector eléctrico de "reaccionaria" ". Según este experto, durante todo el siglo XX los responsables de la toma de

\footnotetext{
una población de unos 30-35 millones de habitantes en su área metropolitana, mientras que al este en la conurbación Kioto, Osaka, Nara y Kobe con los municipios de sus alrededores alcanza los 17 millones, la mitad de habitantes por lo que la demanda de electricidad es menor. La rivalidad entre ambas queda patente en la diferencia de potencia, que se debe a que mientras que unos buscaron ejemplo en Estados Unidos, los otros miraron hacia Europa y más en concreto hacia Alemania.

17 Scalise, Paul J. (2009): The politics of restructuring: agendas and uncertainty in Japan's electricity deregulation, Oxford, Oxford University Press.
} 
decisiones en materia energética han carecido de una estrategia energética integral (comprehensive), por lo que la seguridad energética dependía de una serie de reglas, regulaciones y leyes $a d$ hoc a las que se aferraban en momentos de incertidumbre nacional o crisis económica. Ejemplos de ello son las medidas que se adoptaron en respuesta a las crisis petrolíferas de 1973 y 1979, a la década perdida de 1990 o las iniciativas del nuevo milenio fruto de su compromiso de contribuir a la reducción del calentamiento global. A comienzos del siglo XXI, con su visión a largo plazo, la Ley Básica de Política Energética ${ }^{18}$ de 2002 venía a poner orden en la situación. Repasemos brevemente algunos puntos clave que han conducido a la situación actual.

\subsection{La década de 1970: las Tres Leyes Básicas para la Generación de Electricidad (Dengen sanpo)}

Entre las iniciativas legislativas que más han influido en la trayectoria de la energía nuclear del país están los conocidos como "Dengen Sanpo" o Tres Leyes Básicas para la Generación de Electricidad de 1974, que se fraguaron gracias a la colaboración entre el mundo empresarial, los altos funcionarios y los políticos con el consenso de estabilizar el orden político prevalente y crear un marco institucional en el que encuadrar las decisiones sobre los lugares idóneos para construir centrales nucleares a fin de facilitar la introducción de la energía nuclear ${ }^{19}$.

Según otros autores, el esquema lo que en realidad perseguía era allanar el camino a la construcción de las centrales nucleares mediante la concesión de subsidios y prebendas a los municipios receptores. Los subsidios procedían de un impuesto "invisible"20, que se cobraba a través de la factura eléctrica ${ }^{21}$. Por su parte, los municipios eran cuidadosamente seleccionados entre los que menor oposición mostrarían a la construcción de centrales nucleares en su término.

En su obra Site Fights, Aldrich analiza características en común ${ }^{22}$ : por lo general comunidades rurales donde el nivel de estudios de la población local es inferior y en las que se ha dado una fuerte emigración a la ciudad, por lo que la llegada de inversión de grandes empresas eléctricas se ve como una oportunidad para crear empleo y dinamizar la economía, reteniendo así a la población. Situadas lo suficientemente lejos de grandes núcleos urbanos a los que sirven como para no suponer una molestia, pero no demasiado a fin de no incrementar el coste de la construcción de infraestructuras para el transporte y, posteriormente, del propio transporte. Además, los ayuntamientos suelen presentar déficit por lo que el pago de impuestos beneficiaría a las arcas municipales ${ }^{23}$. Esta descripción es un fiel reflejo de lo que

\footnotetext{
$18 \mathrm{Su}$ texto puede consultarse en los archivos del CNIC (Citizen's Nuclear Information Center), en http://www.cnic.jp/english/newsletter/nit97/nit97articles/nit97enypolicy.html

19 Calder, Kent: “Beyond Fukushima: Japan's Emerging Energy and Environmental Challenges”, Orbis, vol. 57, n³ (abril 2013), p 441.

${ }^{20}$ Aldrich, Daniel P.: "Japan's Nuclear Power Plant Siting: Quelling Resistance", The Asia-Pacific Journal, Vol. 3, Issue 6 (June 13, 2005), en http://japanfocus.org/-Daniel_P_Aldrich/2047/article.html

${ }^{21}$ Aldrich lo denomina invisible porque está planteado de tal manera que muchos japoneses no son conscientes de pagarlo.

${ }^{22}$ Aldrich, Daniel P. (2010): Site Fights: Divisive Facilities and Civil Society in Japan and the West. Ithaca, N.Y., Cornell University Press.

23 Aldrich argumenta que para el año 2002, una comunidad que aceptase que se construyera en su término municipal un reactor de 1,35 millones de kilovatios podría recibir subsidios de hasta 450.000 millones de yen. Aldrich, "Japan's Nuclear Power Plant Siting...”, op.cit.
} 
sucedió en la prefectura de Fukushima, que junto a Fukui y Niigata, es una de las prefecturas que más reactores nucleares llegaron a alojar ${ }^{24}$.

Independientemente del enfoque, estas tres leyes han establecido los parámetros político-económicos dentro de los cuales ha operado la política nuclear civil de Japón desde entonces. En conjunto, las tres leyes establecieron un esquema de incentivos para los municipios de acogida, que por otro lado funcionó tan bien que permitió al Partido Liberal Demócrata perpetuarse en el poder.

La primera ley establecía un sistema impositivo que generaba ingresos, procedentes de un sobrecargo en la factura eléctrica, que estaban destinados a promover la aceptación popular de la energía nuclear así como de otras alternativas al petróleo. La segunda ley creaba una cuenta especial en la que ingresar el montante generado por la primera ley, así los impuestos que los usuarios pagan al gobierno por este concepto van primero a una cuenta general del presupuesto nacional japonés y luego se transfieren a una cuenta especial, desde la que se destinan fondos a proyectos que cumplen los requisitos. La tercera ley regula la utilización de los fondos de la Cuenta Especial para otorgar subsidios y prebendas, tales como carreteras, complejos deportivos o centros cívicos, a los municipios situados en áreas cercanas a nuevas centrales nucleares.

Las leyes eran gestionadas por el MITI (Ministry of International Trade and Industry), el mismo órgano burocrático encargado de la política energética. Con raíces en la administración de posguerra, el precursor del actual METI contaba con amplios poderes regulatorios y se encargaba de administrar los recursos favoreciendo los intereses de las operadoras eléctricas, que a su vez se mostraban receptivos al adoctrinamiento del MITI respecto a las políticas de seguridad energética. Una relación mutuamente beneficiosa.

\subsection{La década de 1980: Marcando las líneas estratégicas de la política energética}

En 1981 Ronald A. Morse, que en ese momento era director del Programa de Asia Oriental del think tank estadounidense Woowdrow Wilson Center for Scholars, afirmaba que si bien Japón había superado las dos crisis petrolíferas que habían marcado la década de 1970, estas habían hecho mella en un país cuyo supervivencia y prosperidad económica dependían del petróleo por lo que comenzaba la década de 1980 resuelto a diversificar sus fuentes de energía a través de la cooperación entre el sector público y el privado ${ }^{25}$.

A raíz de la crisis de 1973-74, el gobierno japonés se ha esforzado por reducir la dependencia y vulnerabilidad del sector energético a fin de garantizar la seguridad energética. Morse resume en cinco puntos los elementos básicos de la política energética de Japón, en la que el gobierno tomaría un papel más activo, y que aún hoy siguen marcando su estrategia:

1. Fomentar el desarrollo de actividades de upstream por empresas japonesas de petróleo en el exterior, así como hacer un mejor uso de las potenciales fuentes energéticas nacionales.

2. Desarrollar a corto plazo alternativas energéticas no basadas en el petróleo: carbón, energía nuclear y gas natural licuado (GNL)

\footnotetext{
${ }^{24}$ En su momento de mayor actividad nuclear en los años 2006-2007, Japón llegó a contar 54 reactores nucleares, más de la mitad de ellos se encontraba en tres prefecturas (el equivalente a nuestras provincias): Fukui alojaba 15 reactores, Fukushima 10 y Niigata 7.

${ }^{25}$ Morse, Ronald A. y University of California, Berkeley (Eds.) (1981): The politics of Japan's energy strategy: resources-diplomacy-security, Berkeley, Institute of East Asian Studies, University of California, pp. 1-3.
} 
3. Diversificar las fuentes de suministro de petróleo y fomentar las negociaciones directas y gobierno a gobierno con las naciones productoras de petróleo.

4. Fomentar la conservación de la energía, así como la comercialización de nuevas tecnologías energéticas

5. Preparar procedimientos para la gestión de emergencias energéticas y almacenar reservas de petróleo a fin de satisfacer las necesidades del país en caso de que se produjese una interrupción grave del suministro.

A juicio de Morse, aunque ya en esta época los japoneses manejaban el concepto de "seguridad energética", aún no lo utilizaban con el mismo enfoque que en Estados Unidos, tampoco eran conscientes de su alcance, ni establecían un vínculo entre "energía" y "seguridad" lo cual se hacía patente en el marco de su estrategia de "seguridad integral"26.

\subsection{La Ley para la Conservación de la Energía}

En 1979 entró en vigor la primera Ley para la Conservación de la Energía, que tenía por objetivo potenciar el ahorro energético en los sectores industrial, comercial, residencial y del transporte. La ley definía un nuevo marco según el cual las empresas deben medir su consumo anual de electricidad y comunicárselo al gobierno. También fijaba estándares de eficiencia para edificios y hogares.

Además, la Ley para la Conservación de la Energía instauró un programa llamado "Top Runner" que fomenta la competencia y la innovación en la fabricación e importación de electrodomésticos, automóviles y otros equipos que suponen el $70 \%$ del consumo doméstico ${ }^{27}$, estableciendo objetivos de eficiencia a medio y largo plazo (3 a 10 años) para fabricantes e importadores, sin que ello conllevase un aumento del precio para los consumidores.

En las tres décadas que lleva en funcionamiento, el programa "Top Runner" ha conseguido excelentes resultados, un ejemplo de ello es el hecho de que los automóviles han ido aumentando paulatinamente su eficiencia, por lo que con la misma cantidad de combustible cada vez se pueden recorrer más kilómetros. Así, según el METI, en 1999 un turismo de tamaño medio era capaz de hacer 16 kilómetros más por litro de gasolina que en 1979, alcanzando los 19,9 kilómetros por litro a principios de la década de 2010.

\subsection{La Ley Básica de Política Energética de 2002 y Nueva Estrategia Nacional de Energía}

Las líneas de acción de la actual política energética japonesa se enmarcan dentro la Ley Básica de Política Energética, ley $N^{\circ} 71$, de 14 de junio 2002, que delineaba las "3 Es" del trilema anteriormente expuesto: la seguridad energética (artículo 2), la sostenibilidad del medio ambiente (artículo 3) y la efíciencia económica (artículo 4).

\footnotetext{
${ }^{26}$ Dadas las limitaciones que le imponía su Constitución pacifista en el plano militar, Japón encontró en la seguridad integral (comprehensive security) un concepto muy útil a la hora de interpretar su política de seguridad. Para ampliar información, véase la reedición del libro de 1983 de Chapman y otros, Chapman, J. W. M., Gow, I. T. M., \& Drifte, R. (2013): Japan's Quest for Comprehensive Security: Defence - Diplomacy Dependence. Bloomsbury 3PL

${ }^{27}$ Según el World Enegy Council, en los países industrializados, los tres sectores que representan el 70\% del consumo total de electricidad son: Motores (45\%), iluminación (15\%), y electrodomésticos y otros aparatos electrónicos (20\%). World Energy Resources: 2013 Survey, en https://www.worldenergy.org/publications/2013/world-energy-resources-2013-survey/
} 
La Ley Básica no establece objetivos cuantificables, sino que en virtud de su artículo 12 faculta al gobierno para formular un plan estratégico de energía que promueva medidas para garantizar un suministro energético que satisfaga las necesidades de la demanda. Dicho plan estratégico debe ser revisado al menos cada tres años y sometido al Gabinete ${ }^{28}$ para su aprobación. El Primer Plan Estratégico de Energía data de 2003 y desde entonces se ha revisado en tres ocasiones: 2007, 2010 y 2014.

\section{Gráfico 4. Planes Estratégicos de Energía 2003-2014}

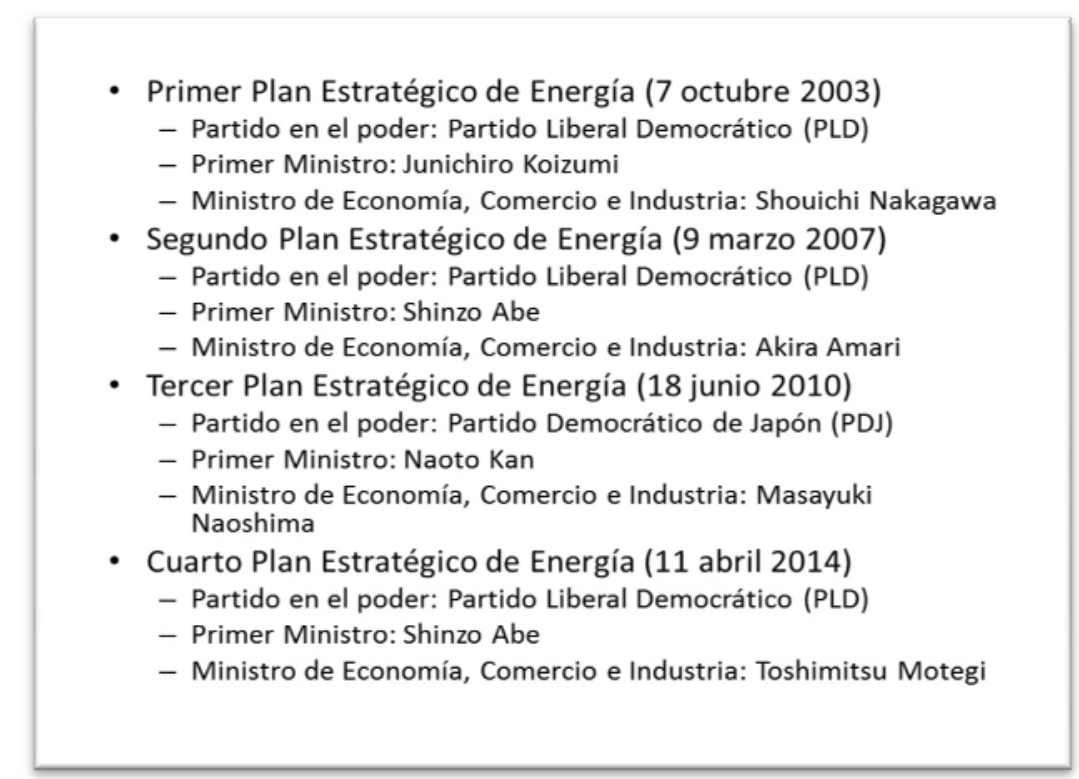

Fuente: METI

La Ley Básica de Energía era la primera ley nacional que abordaba de forma integradora la seguridad energética. La Ley pone el énfasis en el medio ambiente y en asegurar el suministro energético, principios a los que se subordinan cuestiones económicas como la liberalización de los mercados de energía o la desregulación o reforma del sector, entre otras. Además, justificaba la intervención del Estado en el sector eléctrico, tanto a nivel nacional como internacional.

En mayo de 2006, debido a la creciente competencia por los recursos con China y la India, y a un aumento del precio del petróleo crudo importado desde 1998 (12,8 dólares / barril) hasta 2006 (63,5 dólares / barril $)^{29}$ que rondaba el 400\%, el METI elaboró una Nueva Estrategia Nacional de Energía (Shin-Kokkai enerugii senryaku o NNES, por sus siglas en inglés) ${ }^{30}$. El documento parte de la premisa de que los precios del petróleo seguirán siendo elevados tanto a medio como a largo plazo, dado el poco margen de acción existente en el mercado debido a lo ajustada que está la oferta a la demanda.

Además, la Nueva Estrategia situaba por primera vez al GNL en un plano similar al del petróleo al considerar que el GNL es una fuente tan insegura como el propio petróleo. Para garantizar la seguridad energética con un horizonte 2030 se perfilaban una serie de estrategias y objetivos, como la diversificación de proveedores y la conservación de energía, el aumento de la eficiencia energética en al menos un 30\%, disminución de la dependencia del

\footnotetext{
${ }^{28}$ Naikaku, formado por el Primer Ministro y sus ministros.

${ }^{29}$ Datos extraídos de Scalise, op. cit.

${ }^{30}$ El texto de la New National Energy Strategy está disponible en http://www.enecho.meti.go.jp/en/reports/pdf/newnationalenergystrategy2006.pdf
} 
petróleo en un $40 \%$ y otorgar un mayor porcentaje de la energía nuclear en el mix energético llegando a alcanzar el 30\%-40\%. Asimismo, el documento respalda un mayor control sobre los recursos a través de la intervención de empresas japonesas en el upstream, lo cual sorprende a los expertos ${ }^{31}$ dados los malos resultados de $\mathrm{JNOC}^{32}$ que en 2004 se disolvió y fusionó formando JOGMEC (Japan Oil, Gas and Metals National Corporation) ${ }^{33}$.

\subsection{El Tercer Plan Estratégico de Energía (18 junio 2010), revisión 2011}

En septiembre de 2009, Japón anunciaba en unas negociaciones sobre el cambio climático de las Naciones Unidas que se fijaba como meta ${ }^{34}$ reducir en un $25 \%$ las emisiones de gases de efecto invernadero ${ }^{35}$. En consecuencia, en el Plan Estratégico de Energía publicado al año siguiente y único elaborado por el partido de la oposición PDJ hasta el momento, la política del gobierno japonés giraba en torno a la reducción de emisiones de gases de efecto invernadero.

La eficiencia económica y la seguridad energética quedaban así subordinadas a la "E" del medio ambiente (Environment), que vertebraba las previsiones de un mix energético en el que la energía nuclear (en calidad de energía limpia, eficiente y económica) estaba llamada a desempeñar un papel protagonista, que las energías renovables complementarían en un discreto segundo plano.

El Plan fijaba una serie de ambiciosas metas para 2030 como la de elevar al $70 \%$ el porcentaje de energías de emisiones cero, reducir a la mitad las emisiones de $\mathrm{CO} 2$ de los hogares y fomentar la eficiencia energética en el sector industrial, situándolo al más alto nivel mundial. Una serie de medidas específicas, tanto por el lado de la oferta como por el de la demanda, estaban asociadas a estos ambiciosos objetivos. Por el lado de la oferta, el gobierno se proponía por una parte poner en marcha 14 nuevos reactores nucleares hasta $2030 \mathrm{y}$, por la otra, que las energías renovables ampliaran su tasa de mercado en un $10 \%$ gracias a la "Feedin Tariff" (FIT, por sus siglas en inglés), es decir, una retribución fijada por el gobierno para la energía inyectada a la red. Además, contaba con tener construida para la década de 2020 la red interactiva de última generación más avanzada del mundo. Por el lado de la demanda, una de las medidas que pretendía poner en práctica era la de establecer una estructura con bajas emisiones de $\mathrm{CO} 2$ ("low carbon") logrando la mayor eficiencia energética a nivel mundial en el sector industrial, potenciado la reducción de emisiones en hogares y oficinas, y elevando la ventas de automóviles de última generación hasta el 50\% del total para el año 2020 y al 70\% en el 2030, entre otras muchas medidas.

31 Evans, Peter C.: The Brookings Foreign Policy Studies Energy Security Series: Japan, (2006), The Brookings Institution, en http://www.brookings.edu/ /media/research/files/reports/2006/12/japan/12japan.pdf

32 Japan National Oil Corporation (JNOC) constituida por el gobierno japonés y dominó el mercado de hidrocarburos hasta su nacionalización en 2004. Su misión era la de fomentar la prospección y producción petrolífera nacional y exterior. En 2004 se escindió en varias empresas con la excusa (u objetivo, depende de la lectura) con la finalidad de fomentar la competencia en el sector energético. Muchas de sus actividades fueron asumidas por JOGMEC, cuya misión es la de respaldar a empresas japonesas de prospección y explotación de recursos energéticos en el exterior, así como de la gestión de reservas. Otras empresas fruto de la escisión son Japex (Japan Petroleum Exploration Company) e Inpex, que es en la actualidad la mayor empresa de gas y petróleo de Japón.

${ }^{33}$ Para más información sobre esta organización, consúltese su página web: http://www.jogmec.go.jp/english

34 Aunque la meta estaba subordinada al resultado final de la negociación, una reducción así dificultaría la consecución de las 3Es, puesto que su eficiencia energética es de por sí bastante elevada hasta el punto de que cualquier reducción marginal resultaría cara.

${ }^{35}$ McCurry, Justin: “Japan's new prime minister promises ambitious greenhouse gas cuts", The Guardian, 7 de septiembre de 2009, en http://www.theguardian.com/environment/2009/sep/07/japan-greenhouse-gas-cuts 
Este Plan era el que estaba vigente a principios de 2011, en el momento del accidente nuclear de Fukushima. La energía nuclear representaba entonces casi el 30\% de la producción eléctrica total del país, esperándose que en 2017 se convirtiese en el 41\% y llegase al 50\% en 2030. Sin embargo, tras el accidente de Fukushima, el gobierno del PDJ dio un giro radical al fijarse como objetivo el abandono total del modelo nuclear.

El Primer Ministro Naoto Kan, que ocupaba el puesto en el momento del accidente nuclear de Fukushima, había optado por cerrar las centrales nucleares tras el accidente y se había declarado partidario de abandonar la energía nuclear. La decisión de cerrar las centrales nucleares no complació al poderoso lobby nuclear, que durante años había cultivado una relación de connivencia con el Partido Liberal Democrático ${ }^{36}$. Kan pertenecía al Partido Democrático de Japón (PDJ), que ha estado en la oposición durante toda la posguerra y había desbancado a su eterno rival el Partido Liberal Democrático (PLD) en 2009. El desgaste político por su criticada gestión de la crisis del accidente nuclear, hizo que el PDJ fuese incapaz de revalidar su gobierno en las elecciones de 2012.

\subsection{La Estrategia Innovadora para la Energía y el Medio Ambiente}

Unos meses antes elecciones de las elecciones de diciembre de 2012, el PDJ se apresuró a pasar una estrategia energética que guiara al país por la senda de las desnuclearización, hacia derroteros más sostenibles y alejados de las tradicionales conexiones de la "aldea de la energía nuclear" "37. Se trataba de la Innovative Strategy for Energy and the Environment de septiembre de $2012^{38}$, que pronto quedó olvidada pues tras ganar las elecciones el PLD, con Shinzo Abe al frente, retomó la senda nuclear.

La Estrategia Innovadora, que reposaba sobre tres pilares, perseguía reducir la dependencia tanto de la energía nuclear como de los combustibles fósiles, maximizando la "energía verde" y potenciando por una parte la eficiencia energética y, por la otra, las energías renovables. La nueva estrategia también revisaba los objetivos de emisiones de CO2 para 2030: Si pre-Fukushima perseguían una reducción del 30\% de las emisiones, en relación al nivel de 1990, con la nueva estrategia la reducción descendía al $20 \%$ en caso de que el crecimiento económico fuese moderado y al $10 \%$ en caso de que fuese mayor ${ }^{39}$.

El primer pilar consistía en construir una sociedad que no dependiese de la energía nuclear a la mayor brevedad posible, para lo cual movilizarían todos los recursos políticos a su alcance a fin de lograr el objetivo de que las centrales nucleares dejasen de funcionar por completo en 2030. Para ello, no se construirían los nuevos reactores nucleares que estaba previsto construir, ni se alargaría la vida de los reactores más allá de los 40 años.

\footnotetext{
${ }^{36}$ La relación entre el lobby nuclear y el partido gobernante se veía reforzada mediante dinámicas de "puertas giratorias" (en japonés amakudari, literalmente descenso del cielo), también la prensa se ha hecho eco de las donaciones hechas por altos cargos de TEPCO (la empresa propietaria de la central nuclear de Fukushima) al PLD. Para más información véase, Casado, María F.: "Fukushima: un desastre nuclear evitable", Esglobal, 10 de marzo de 2015, en http://www.esglobal.org/fukushima-un-desastre-nuclear-evitable/

37 Aldea de la energía nuclear o genshi ryoku mura, conocido en inglés como nuclear village, es el triángulo de hierro en el que se mueven una serie de intereses creados entre las poderosas empresas del sector eléctrico, los altos funcionarios y los políticos, con su capacidad de influencia sobre medios de comunicación y círculos académicos. Para más información véase, Casado, María F.: "Fukushima: un desastre nuclear evitable", Esglobal, 10 de marzo de 2015, en http://www.esglobal.org/fukushima-un-desastre-nuclear-evitable/

${ }^{38}$ Ministry of Economy, Trade and Industry (2012): Innovative Strategy for Energy and the Environment, en http://www.un.org/esa/socdev/egms/docs/2012/greenjobs/enablingenvironment.pdf

39 "Innovative Strategy to end nuclear", World Nuclear News, 12 de septiembre de 2012, en http://www.worldnuclear-news.org/NP Innovative strategy to end Japanese nuclear 1409121.html
} 
El segundo pilar consistía en poner en marcha la "Revolución de la energía verde", estableciendo un nuevo marco en el que participasen todas las partes interesadas, incluidos los consumidores. Fomentando la participación social, se pretendía estabilizar el abastecimiento energético y la conservación del medio ambiente, a la vez que se propiciaba la aparición de nuevas actividades económicas que dinamizasen la economía local.

El tercer pilar, relacionado con los dos anteriores, tenía por objeto garantizar un suministro estable de energía, tomando como base los combustibles fósiles pero haciendo un uso más eficiente, y acelerando la investigación y desarrollo de las tecnologías energéticas del futuro.

\subsection{El Cuarto Plan Estratégico de Energía y el Libro Blanco de la Energía}

En junio de 2014 se publicó el Libro Blanco de la Energía 2013, precedido en marzo de 2014 por el Cuarto Plan Estratégico de Energía ${ }^{40}$ (enerugi kihon keikaku) con un horizonte 2030 que dejaba por concretar el futuro mix energético de Japón. A finales de abril de 2015, tras un largo periodo de reflexión, el METI hacía públicos estos datos que dejarán notar su influencia tanto en la futura seguridad energética de Japón, como en la de las emergentes economías asiáticas y en el panorama energético internacional.

En cuanto a las líneas del Cuarto Plan Estratégico de Energía, la nueva política energética de Japón tiene como objetivo superar los retos que se le plantean reduciendo simultáneamente los costes de generación y compra de energía primaria, distribución y consumo. El Plan también revalida su compromiso con el " $3 \mathrm{E}+\mathrm{S}$ " en su capítulo segundo, sección primera, y establece como metas clave equilibrar el mix energético fortaleciendo el papel de las energías renovables y no convencionales, diversificar fuentes apartándose del petróleo mediante una reducción de su uso en el sector transporte ${ }^{41}$ y potenciar el desarrollo tecnologías punta de generación de electricidad a partir de combustibles fósiles, más eficientes y menos contaminantes que las actuales.

En cuanto a la generación de electricidad, el Plan apuesta por diversificar las fuentes energéticas primarias, por una parte favoreciendo la introducción de energías renovables a través de la desregulación o reforma del sector eléctrico y, asimismo, fomentando la adopción de energías renovables como la geotérmica o la eólica mediante la implantación de redes inteligentes. El actualmente gobernante Partido Liberal Democrático es pro-nuclear, por lo que la energía nuclear está llamada a ocupar un puesto en su política energética, que tal vez no sea tan preeminente como el que se planteaba antes del accidente nuclear de Fukushima, pero no desaparece del mapa.

No obstante, antes de volver a poner las centrales nucleares en funcionamiento, estas deberán pasar las pruebas pertinentes dentro del examen que está realizando la nueva e independiente NRA. Otro de los esfuerzos es el de introducir centrales térmicas (de carbón y

\footnotetext{
${ }^{40}$ El documento en su versión inglesa está disponible en la página web de la Agency for Natural Resources and Energy, que forma parte del METI, en la siguiente dirección: http://www.enecho.meti.go.jp/en/category/others/basic_plan/pdf/4th_strategic_energy_plan.pdf

${ }^{41}$ El sector transporte es uno de los que más consume. A través de subsidios, el gobierno fomenta la adopción de tecnologías disruptivas como las baterías de hidrógeno (fuel cells) y las baterías eléctricas (electric batteries), como son las de ion-litio. Estos dos modelos son los que están luchando en la actualidad por hacerse con el mercado de vehículos utilitarios. La batalla está abierta en varios frentes como el precio o la capacidad de hacer frente a la demanda del mercado con una producción suficiente de automóviles, sin embargo el factor que más "ansiedad" provoca es la autonomía de conducción por carga, dado que la infraestructura de recarga se encuentra aún en una fase incipiente, muy lejos aún de la ubicuidad con que se encuentran las gasolineras.
} 
GNL) más eficientes y menos contaminantes. En este apartado, otra de las líneas de acción es la de diversificar las fuentes de aprovisionamiento de energía primaria, para lo cual se están buscando nuevos proveedores de GNL a fin de reducir los costes y, por otra parte, se está invirtiendo en $\mathrm{I}+\mathrm{D}+\mathrm{i}$ para desarrollar fuentes propias de energía, tales como la solar $^{42}$ o los hidratos de metano, sobre los que trataremos más adelante. Un claro ejemplo de ello es que en el verano de 2015, cuando los ministerios sometieron sus peticiones de presupuesto para el año fiscal 2016 (del 1 de abril de 2016 al 31 de marzo de 2017) el Ministerio del Medio Ambiente (MOE) incrementó su solicitud de fondos en un $62 \%$ con respecto al año fiscal 2015 atribuyendo el aumento a la necesidad de financiar proyectos de energía renovable y eficiencia energética ${ }^{43}$.

También en el sector de la distribución se inscribe la reforma del mercado eléctrico que tiene la triple finalidad de llevar a cabo la desagregación o separación de las actividades de generación, transmisión y venta de electricidad, la total liberalización de las actividades de generación de electricidad y venta al por menor y, por último, la puesta en funcionamiento de una red eléctrica nacional que permita trasladar la electricidad de un lado a otro del país cuando sea necesario.

Por último en el sector consumo, los esfuerzos se centrarán en hacer más eficiente a la industria mediante el uso de tecnología punta que fomente un uso más eficiente y competitivo de la energía, una mayor conservación de la energía, incluido el programa "Top Runner"44, y por último fomentar programas de gestión eficiente de la energía como los sistemas de demanda-respuesta (redes inteligentes).

\section{Actual situación energética}

El actual nivel de dependencia de los minerales fósiles es comparable al que Japón tenía a comienzo de la década de 1970. Si antes de que la crisis de 1973 provocase su transición energética hacia la energía nuclear, el $89,7 \%$ de sus fuentes de energía primaria procedían de los combustibles fósiles $(75,5 \%$ del petróleo y $16,9 \%$ del carbón, porcentaje este último del que $4,3 \%$ de producción nacional), un año después del accidente nuclear de Fukushima, en 2012, la dependencia de los combustibles fósiles llegaba al 92\%. Según los datos de la International Energy Agency (IEA), Japón es el mayor importador neto de GNL, el segundo mayor importador neto de carbón y el tercero de petróleo en crudo y derivados del petróleo, por detrás de China y Estados Unidos.

\footnotetext{
${ }^{42}$ Véase este artículo científico sobre los proyectos de investigación para la construcción de una central solar en el espacio. Sasaki, Susumu: "How Japan plans to build an orbital solar farm", IEE Spectrum, 24 de abril de 2014, en http://spectrum.ieee.org/green-tech/solar/how-japan-plans-to-build-an-orbital-solar-farm

${ }^{43}$ DeWit, Andrew: "Japan's Bid to Become a World Leader in Renewable Energy", The Asia-Pacific Journal: Japan Focus, vol. 12, no 39-2 (septiembre 2015), en http://apjif.org/-Andrew-DeWit/4385

${ }_{44}$ Este programa estaba dirigido inicialmente a las empresas con el objetivo de que empleasen equipos de bajo consumo. En los hogares se traduciría, por ejemplo, en la utilización de bombillas de bajo consumo y led.
} 
Gráfico 5. Diez mayores importadores netos de GNL, petróleo en crudo y carbón.

\begin{tabular}{|l|r|}
\hline GNL (2013) & \multicolumn{1}{c|}{ Mt } \\
\hline Japón & 123 \\
\hline Alemania & 76 \\
\hline Italia & 62 \\
\hline Corea del Sur & 53 \\
\hline China & 49 \\
\hline Turquía & 45 \\
\hline Francia & 43 \\
\hline Reino Unido & 39 \\
\hline EE.UU. & 37 \\
\hline España & 30 \\
\hline Otros & 279 \\
\hline Total & 836 \\
\hline
\end{tabular}

\begin{tabular}{|l|r|}
\hline $\begin{array}{c}\text { Petróleo crudo } \\
(2012)\end{array}$ & $\mathrm{Mt}$ \\
\hline EE.UU. & 442 \\
\hline China & 269 \\
\hline India & 185 \\
\hline Japón & 179 \\
\hline Corea del Sur & 128 \\
\hline Alemania & 93 \\
\hline Italia & 74 \\
\hline España & 60 \\
\hline Países Bajos & 57 \\
\hline Francia & 57 \\
\hline Otros & 507 \\
\hline Total & 2051 \\
\hline
\end{tabular}

\begin{tabular}{|l|r|}
\hline Carbón (2013) & Mt \\
\hline China & 320 \\
\hline Japón & 196 \\
\hline India & 178 \\
\hline Corea del Sur & 127 \\
\hline Taipei, China & 68 \\
\hline Alemania & 50 \\
\hline Reino Unido & 49 \\
\hline Turquía & 28 \\
\hline Malasia & 23 \\
\hline Italia & 20 \\
\hline Otros & 211 \\
\hline Total & 1270 \\
\hline
\end{tabular}

Fuente: International Energy Agency: 2014

Por otra parte, en 2014 Japón habría sido el tercer mayor consumidor mundial de petróleo, según el informe sobre Japón publicado en enero de 2015 por la Energy Information Administration estadounidense, por detrás de Estados Unidos y China, aunque la demanda de petróleo ha descendido un $22 \%$ como media desde 2000 . Un descenso que la EIA achaca a factores estructurales como la substitución de combustibles (preferencia por el GNL) en línea con su transición energética y sus metas medioambientales, el descenso de la población y los porcentajes de eficiencia energética fijados por el gobierno.

\subsection{Combustibles fósiles: GNL (Gas Natural Licuado), petróleo y carbón.}

Tras el accidente nuclear de Fukushima, petróleo y GNL han ocupado el lugar de la energía nuclear en la generación de electricidad. Como hemos visto, Japón se ha convertido en el mayor consumidor mundial de GNL y tiene visos de seguir siendo uno de los principales consumidores a corto y medio plazo. Debido al cierre paulatino de las centrales nucleares japonesas, la demanda de GNL aumentó en un 30\% 1legando a ascender a 90 millones de toneladas en el año fiscal $2012^{45}$, frente a los 70 millones de 2010.

El GNL presenta un riesgo geopolítico moderado en comparación al petróleo y es el combustible fósil cuyas emisiones de $\mathrm{CO} 2$ son menores, razón por la cual se ha convertido en una fuente importante de energía a medio plazo y también podría convertirse en un pilar de una sociedad del hidrógeno. Además, con la competencia creciente del shale gas gas (también conocido como gas de esquisto o gas pizarra $)^{46}$ y la diversificación de proveedores, su precio podría reducirse.

\footnotetext{
${ }^{45}$ Los años fiscales comienzan en abril y finalizan en marzo. El año fiscal 2012, por tanto, va de abril de 2012 a marzo de 2013

${ }^{46}$ El shale gas es gas natural, como el que actualmente se utiliza en muchos hogares y como repostaje de autobuses, taxis y automóviles. Se considera un gas no convencional porque en su extracción se utilizan técnicas distintas a las tradicionales (es decir, técnicas no convencionales como el fracking o fracturación hidraulica) ya que se halla en formaciones rocosas y arcillosas de muy baja permeabilidad.
} 
Por su parte, el petróleo sigue siendo una importante fuente energética de la que dependen el sector de los transportes, la industria petroquímica y las centrales térmicas. Entre sus desventajas se encuentran la alta volatilidad de sus precios, la sensibilidad a las tensiones geopolíticas y el hecho de que el mercado está controlado por los productores. Mientras que el carbón ha cobrado importancia en el contexto post-Fukushima dadas su estabilidad y eficacia en relación a su coste, aunque en contrapartida sus emisiones de $\mathrm{CO} 2$ son mayores, un efecto que Japón intenta atenuar recurriendo a tecnologías térmicas más eficientes.

En la antesala de la Cumbre del Clima, celebrada en París en diciembre de 2015, una de las críticas contrarias a Japón era su intención de construir más 40 nuevas centrales térmicas a base de carbón en los próximos años, algunas ya en construcción mientras que otras tan sólo están planificadas. Japón argumenta que el carbón está barato y sus reservas se encuentran en países con menor riesgo geoestratégico, además la tecnología que utiliza es de última generación y menos contaminante ${ }^{47}$. En las conversaciones previas a la cumbre celebradas en Bonn, Alemania, a principios de junio de 2015 la ONG Climate Action Network concedió a Japón el premio a "Fósil del Día" (Fossil of the Day) por el esfuerzo realizado para bloquear el progreso en la acción climática ${ }^{48}$.

\section{Gráfico 5. Origen de las importaciones de crudo 2014}

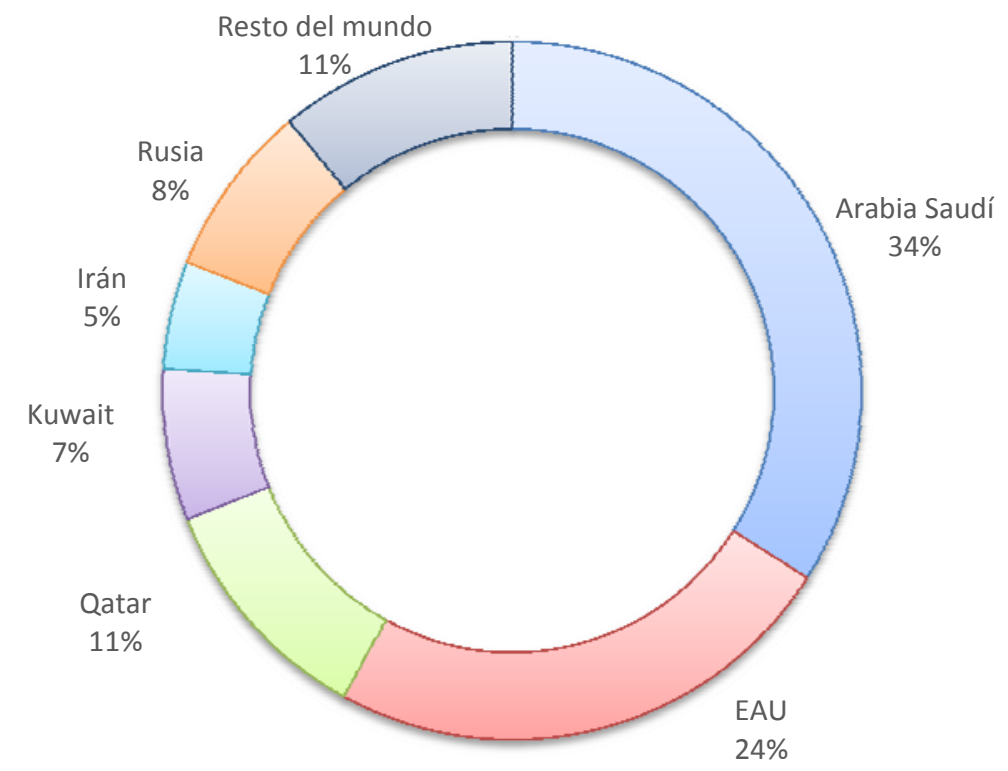

Fuente: METI

El coste del petróleo supone un freno para la economía japonesa, hasta el punto de que en 2011 se produjo el primer déficit comercial desde 1980 y este siguió creciendo en años posteriores. No obstante, la bajada de los precios del petróleo en los mercados mundiales a finales de 2014, que tiene visos de prolongarse a medio plazo, ha propiciado una mejora de la economía japonesa. Tanto para los japoneses de a pie, como para las empresas la bajada a

\footnotetext{
${ }^{47}$ Gracias al fomento de las tecnologías de bajas emisiones (Low-Carbon) para centrales térmicas a base de carbón por parte del gobierno japonés, este tipo de centrales eléctricas ha alcanzado la mayor eficiencia a nivel mundial. Además, por su gestión inteligente, dicha eficiencia se mantiene durante periodos prolongados.

${ }^{48}$ Kurtenbach, Elaine: “At G-7, Japan's energy plan is not all that green”. Phys.org (5 de junio de 2015), en http://phys.org/news/2015-06-g-japan-energy-green.html
} 
corto y medio plazo del coste del combustible y la electricidad han supuesto un respiro. Los expertos prevén que con mayor renta disponible por el ahorro, aumentará el consumo privado, se incrementarán los beneficios de las empresas y sus inversiones empresariales, y también lo harán la oferta y la demanda, llegando incluso a la bajada de precios ${ }^{49}$.

No obstante, para el gobierno japonés es una prioridad reducir los precios de la energía primaria o energía bruta. Por ello, en su Plan Estratégico de Energía 2014 ha definido unas líneas de acción dirigidas a conseguir GNL a precios más competitivos. Japón es consciente de que Rusia, uno de sus principales proveedores de GNL, utiliza los gaseoductos como un arma diplomática que le sirve para acercar posiciones con otros países, entre ellos China. Un instrumento que in extremis podría convertirse en arma arrojadiza. Por ello, por el lado de la oferta ha optado por diversificar sus proveedores (Canadá, Mozambique, Estados Unidos) y acelerar los proyectos upstream gestionados por empresas japonesas, mientras que por el lado de la demanda busca compensar sus necesidades energéticas recurriendo a otros tipos de energía primaria: nuclear (los reactores nucleares están pasando un minucioso proceso de evaluación y podrían volver a funcionar, una vez aprobado), carbón (centrales térmicas más eficientes y limpias) e hidratos de metano (aceleración de la investigación de reservas y tecnologías de extracción).

Abundando en lo expuesto en el párrafo anterior y a modo de ejemplo, una de las líneas de acción es la de fomentar la cooperación público-privada y hacer uso de la "diplomacia de recursos" multinivel con países exportadores de recursos naturales como Canadá, donde grandes empresas japonesas como Mitsubishi Corporation, JAPEX e INPEX ${ }^{50}$ están llevando a cabo una serie de proyectos de extracción de shale gas con visos a su exportación a mercados de Asia-Pacífico que comenzaría en torno a 2018.

La estrategia que persigue el gobierno japonés con estos movimientos es clara: busca diversificar los flujos comerciales actuales aumentando el suministro de GNL y shale gas a fin de presionar a los productores tradicionales de gas convencional y propiciar un cambio de los mecanismos tradicionales de fijación de precios. La "mano invisible" que mueve los hilos del mercado, queda aquí al descubierto: a medida que crezca la oferta, será mayor la capacidad de acción de la demanda.

Por otro lado, otra forma de reducir la dependencia externa es continuar con las exploraciones en su propio territorio, lo cual hasta el momento no ha dado grandes frutos. La mayor parte de sus yacimientos se encuentra en la costa occidental, también se han encontrado depósitos de petróleo y gas natural en el Mar de China Oriental, que no pueden explotarse debido a que es una zona sobre la que mantiene disputas territoriales con China. En 2008, ambas naciones llegaron a un acuerdo para explorar y explotar conjuntamente dos yacimientos $^{51}$, sin embargo el acuerdo no ha dado frutos a causa de la tensa relación entre ambas. Una relación que en los últimos años pasa por sus peores momentos y que podría lugar a una escalada de tensiones en la zona. Las acciones unilaterales que cada estado ha tomado son indicativas del nivel al que han llegado: Por una parte, en 2012 un particular japonés compró unos islotes pertenecientes a las disputadas Senkaku/Diaoyou, China a su vez

\footnotetext{
${ }^{49}$ Nagata, Kazuaki: “Lower oil prices spell good news for Japanese economy”, The Japan Times Online, 12 de enero 2015, en http:/www.japantimes.co.jp/news/2015/01/12/business/economy-business/lower-oil-prices-spellgood-news-japanese-economy/

${ }_{50}$ Japex e Inpex son dos empresas escindidas de JNOC (Japan National Oil Company), privatizada en 2004.

${ }^{51}$ Watts, Jonathan: "China and Japan agree on joint gas exploration of East China Sea", The Guardian, 18 de junio de 2008, en http://www.theguardian.com/world/2008/jun/18/china.japan
} 
instaló una plataforma de producción en la zona disputada y declaró que establecía una zona de defensa aérea que cubría gran parte del Mar de China Oriental.

\subsection{La energía nuclear}

A falta de recursos energéticos propios, la energía nuclear ha sido para Japón una fuente casi autóctona, que además de asegurar un suministro constante para satisfacer la demanda de electricidad de hogares, oficinas e industria, le había permitido cumplir con su compromiso medioambiental de reducción de emisiones de $\mathrm{CO} 2$, y todo ello a un precio supuestamente económico $^{52}$. Japón cuenta con facilidades para reprocesamiento de combustible nuclear en la Planta de Reprocesamiento de Rokkasho, por lo que podría producir electricidad para varios años sin tener que importar.

Hasta el accidente nuclear de Fukushima de marzo de 2011, Japón era el tercer mayor consumidor de energía nuclear a nivel mundial por detrás de Estados Unidos y Francia. La energía nuclear, que representaba aproximadamente una tercera parte del mix energético (27\%) estaba llamada a desempeñar un papel cada vez más importante en el futuro energético de Japón llegando a suponer un 50\% en 2030, según constaba en el Plan Estratégico de Energía que el gobierno había publicado apenas un año antes del accidente, en 2010. Tras el triple desastre, en los años 2012 y 2013 la energía nuclear no ha supuesto más que el 1\% del total.

El accidente supuso un giro inesperado del destino, reavivando el debate en torno a la seguridad de la energía nuclear entre sus defensores y detractores, el panorama se plantea aún incierto basculando entre la oposición mayoritaria de la opinión pública, el activismo de la sociedad civil y el empuje del gobierno y del poderoso lobby de la electricidad por volver a poner en funcionamiento los reactores que gradualmente se detuvieron en frío por labores de mantenimiento, el último de ellos a comienzos de 2012. La única excepción se produjo en julio de 2012, para hacer frente a la demanda del verano y el invierno, el gobierno permitió que dos reactores ${ }^{53}$ funcionaran temporalmente hasta septiembre desde 2013. De ahí el 1\% que apuntábamos en el párrafo anterior.

Tras ganar las elecciones de diciembre de 2012, el recién elegido gobierno del PLD dejó clara su intención de dar un giro a la política energética de Japón, dejando de lado la apuesta antinuclear del gobierno precedente. En virtud de la nueva normativa de seguridad nuclear, Japón planea reiniciar los reactores nucleares que superen los exhaustivos exámenes de la NRA, que comenzó a recibir solicitudes tras su creación en julio de 2013. La NRA se ha caracterizado por ser un ente abierto, más transparente y participativo que su predecesora a la hora de establecer la Nueva Regulación de Seguridad Nuclear, que se publicó y entró en vigor en julio de 2013, y para su elaboración contó con la colaboración de expertos del mundo académico, sin dejar de tener en cuenta la opinión de las compañías eléctricas.

Para que los reactores puedan volver a ponerse en marcha, además de superar las pruebas de resistencia de la NRA, deben recibir el visto bueno tanto de las autoridades locales como de los residentes del municipio donde se encuentre la central. De los 43 reactores nucleares con que cuenta Japón, tan solo 2 se encuentran operativos: Sendai 1 y 2; mientras que otros 23 reactores esperan su aprobación por parte de la NRA, de las autoridades locales o

\footnotetext{
52 En el coste final de la energía nuclear habría que incurrir tanto los subsidios estatales, como las compensaciones derivadas del accidente nuclear.

${ }^{53}$ En concreto los reactores 3 y 4 de la Central Nuclear de Ohi, perteneciente a Kansai Electric Company (KEPCO).
} 
bien han interpuesto recurrido las demandas que les han sido interpuestas por tribunales locales $^{54}$. Finalmente, cinco reactores nucleares cerraron definitivamente en 2015 (Genkai 1, Shimane 1 , Mihama 1 y 2, y Tsuruga ${ }^{55}$, mientras que a finales de marzo de 2006 Shikoku Electric Power Company anunció el desmantelamiento de su reactor nuclear Ikata 1 debido a que ya había alcanzado el final de su vida útil, es decir, 40 años ${ }^{56}$.

En agosto de 2015, en medio de la polémica y la oposición de una parte de la sociedad civil, se puso en marcha y se reconectó a la red una vez hubo alcanzado plena potencia el reactor número 1 de la central nuclear de Sendai, que fue seguido por el reactor número 2 en octubre. Ambos propiedad de Kyūshū Electric Power Company.

También se pusieron en funcionamiento los reactores 3 y 4 de la central nuclear de Takahama, a finales de enero y febrero de 2016 respectivamente, aunque por breve tiempo pues una orden judicial obligó a su cierre el 10 de marzo de 2016. La demanda fue interpuesta ante los tribunales locales por un grupo de activistas antinucleares que ponían en duda los estándares sísmicos y nuevas normas de seguridad. La empresa propietaria y operadora de la central nuclear -Kansai Electric Power Company- recurrió la sentencia una semana después.

Por otro lado, debido a la fuerte oposición pública, un tribunal local denegó el permiso para poner en funcionamiento los reactores 3 y 4 de la central nuclear de Ohi, prefectura de Fukui, pertenecientes también a Kansai Electric Power Company. La operadora también ha recurrido esta sentencia.

En el polo opuesto, Shikoku Electric Power Company ha recibido el visto bueno de la ciudad de Ikata y de la prefectura de Ehime, por lo que espera poner en marcha el reactor 3 de la central nuclear de Ikata en agosto de 2016.

\subsection{Las energías renovables}

Es de prever que tras Fukushima, Japón potenciará las energías renovables y pondrá mayor énfasis en la eficiencia energética. A corto plazo, su recurrencia al GNL y al carbón ha minado su empeño en reducir las emisiones de gases de efecto invernadero.

En la actualidad, la mayor contribución a las energías renovables proviene de la energía hidroeléctrica (en torno al 8-9\%), mientras que en su conjunto el resto de renovables tan solo suponían un 1,6\% dentro del mix energético. La energía solar lleva el liderazgo dentro del grupo de las renovables gracias a iniciativas gubernamentales como el Esquema de Compra del Superávit Residencial de Energía Fotovoltaica puesto en marcha en noviembre de 2009 y el Esquema de Tarifas de Energía Inyectada a la Red (Feed-in Tariff) introducido en 2012, con vistas a incrementar la penetración de las renovables. Si bien el Esquema es visto con recelo porque los precios de compra son caros, ya que están basados en la inversión realizada por los productores individuales y ello repercute en la factura eléctrica de los consumidores finales.

\footnotetext{
${ }^{54}$ Fuente: Japan Nuclear Update, Nuclear Energy Institute, en http://www.nei.org/News-Media/News/JapanNuclear-Update, consultado el 4 de abril de 2016.

55 JAIF: "JAIF Annual Report: World Has 434 Operating NPPs With Capacity of 399GWe", en http://www.jaif.or.jp/en/jaif-annual-report-world-has-434-operating-npps-with-capacity-of-399gwe/

${ }^{56}$ JAIF: Ikata 1 to be decommissioned", en http://www.jaif.or.jp/en/ikata-1-to-be-decommissioned-foreconomic-reasons/
} 


\section{Gráfico 6. Energías renovables, excluyendo hidroeléctrica (porcentaje del mix energético)}

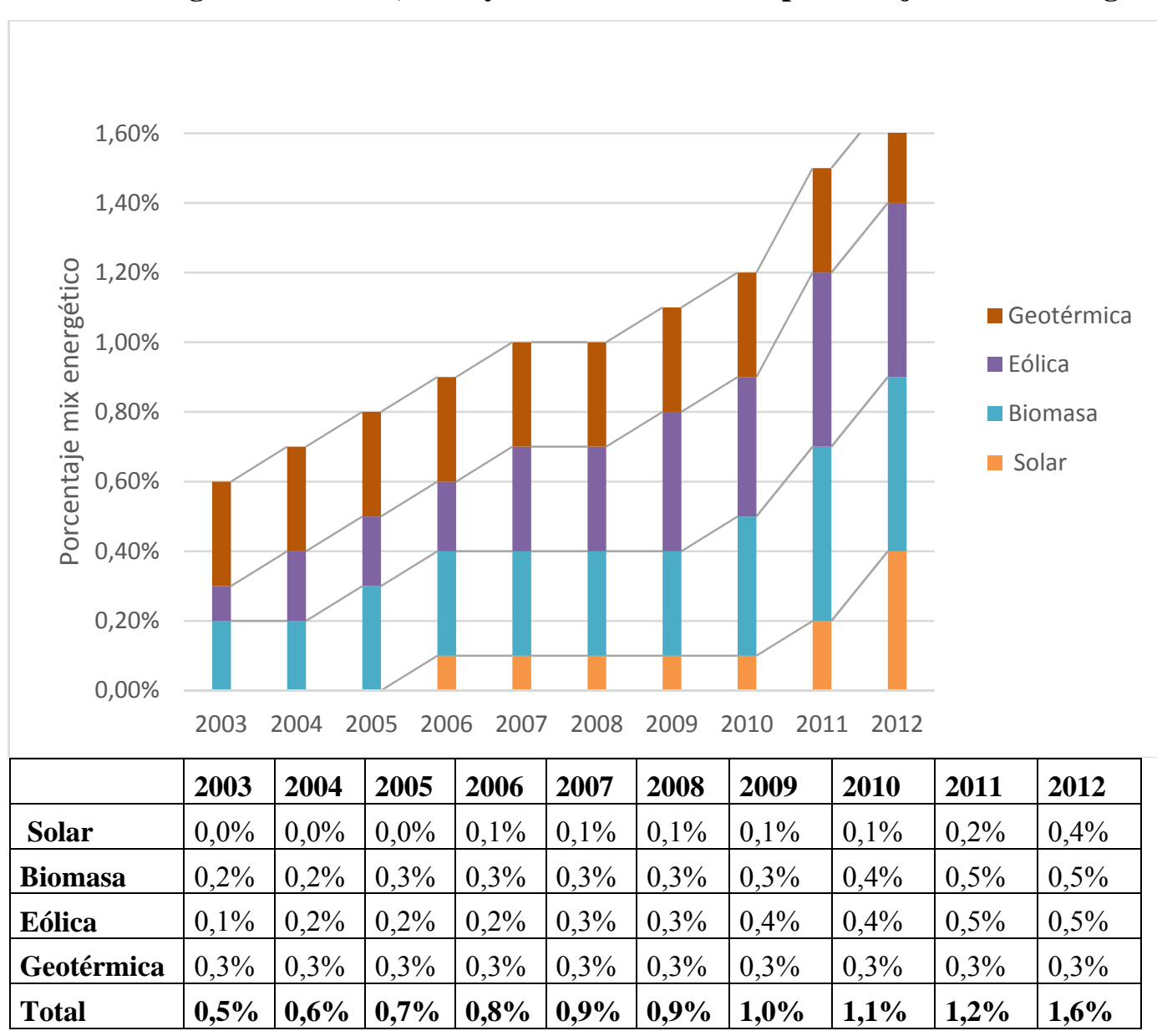

Fuente: METI

La energía solar experimenta en la actualidad un fuerte crecimiento a nivel mundial, debido en parte a la rápida disminución del coste de fabricación de paneles solares y a los subsidios gubernamentales. Los expertos predicen que aproximadamente en una década la curva ascendente de los precios del petróleo se cruzará con la curva descendente del coste de fabricación de los paneles solares, que habrán alcanzado un grado de perfeccionamiento tecnológico que mejorará su eficiencia.

La energía solar presenta entre sus beneficios su rápida instalación y las posibilidades que brinda a la hora de dotar de electricidad a áreas remotas, mientras que entre sus desventajas se encuentran la intermitencia del suministro y los retos que plantea su integración a la red. España ha perdido su liderazgo en este sector dejando de ocupar los primeros puestos mundiales, que en estos momentos se reparten entre Japón, China, Estados Unidos, Alemania e Italia, según estimaciones de Bloomberg para 2013.

El gobierno japonés ha promovido el uso de la energía solar a través de subsidios y de la introducción de la tarifa de inyección a la red (Feed-in Tariff). Antes de su lanzamiento, los hogares eran los principales usuarios de este tipo de electricidad, que posteriormente también han adoptado algunas empresas. Los proyectos aprobados desde 2012 alcanzan la cifra de 79.760 megawatios. No obstante, los avances se podrían ver afectados por las reticencias del gobierno, que parece querer dar marcha atrás presionado por los poderosos lobbies eléctricos. 
La capacidad de la energía eólica mundial se ha duplicado aproximadamente cada tres años y medio desde 1990. A pesar de que sus costes de generación son moderados en comparación a los de la energía hidroeléctrica, son necesarias economías de escala para que resulte rentable. En Japón, según datos del METI, el 66\% de las zonas en las que el viento alcanza una velocidad de más de 6,5 metros por segundo -la velocidad a partir de la cual es económicamente rentable- se concentran al norte, en la región de Tohoku y en la isla de Hokkaido. Los proyectos aprobados en Japón desde 2012 alcanzan la cifra de capacidad de 2.330 megawatios.

Por su parte, la biomasa -también denominada "bioenergía"- es una categoría de combustibles energéticos fabricados a partir de una variedad de materias primas de origen biológico utilizando diversas tecnologías de conversión para generar electricidad,. Desde 2012 los proyectos de biomasa aprobados alcanzan la capacidad de 2.680 megawatios.

\subsection{Futuras fuentes y tecnologías energéticas}

En su esfuerzo por asegurar la independencia energética de Japón, el gobierno japonés está potenciando los proyectos de $\mathrm{I}+\mathrm{D}+\mathrm{i}$ de tecnologías asociadas a la producción de energía y nuevas fuentes de energía, como los hidratos de carbono, cuyos avances revisamos a continuación. Algunas de las nuevas tecnologías están relacionadas con iniciativas como las de la "Sociedad de bajas emisiones de carbono" (Low Carbon Society) o la de la "Sociedad del Hidrógeno" 57 , delineada en el último Plan Estratégico de Energía, en el que se deja vislumbrar una futura sociedad en la que se utilice hidrógeno en la vida diaria y en las actividades industriales. El hidrógeno es un gas que hay que manejar con mucho cuidado, sin embargo es muy eficiente desde el punto de vista energético y no emite gases de efecto invernadero. Revisamos también a continuación cómo su aplicación al transporte.

\section{Hidratos de metano}

Informalmente conocidos como "hielo de metano" o "hielo ardiente", los hidratos de metano son un hidrocarburo formado por cristales de hielo con gas metano natural encapsulado en su interior. Aunque han tardado en entrar en el panorama energético y aún se encuentran en fase embrionaria, su descubrimiento no es reciente pues ya eran conocidos en círculos científicos en el siglo XIX. La URSS se convirtió en 1960 en la primera nación que llevaba a cabo un amplio estudio sobre el terreno al realizar prospecciones en Siberia, con las que se logró confirmar diez años más tarde la existencia de hidratos de metano en el lecho del Mar Negro. En marzo de 2013, Japón se convertía en el primer país que conseguía extraer de forma segura y continua durante varios días hidratos de metano del lecho marino, en unas perforaciones realizadas desde barcos en la costa sur de la isla de Honshu (a unos $200 \mathrm{~km}$ al sur de Tokio), concretamente en la Fosa de Nankai. El éxito se repetía en diciembre de 2014, esta vez en la costa norte. Ambos frutos del trabajo de investigación realizado por la compañía Japan Oil y sufragado por el gobierno japonés, que está apostando fuerte por energías alternativas. De hecho, las perforaciones continúan, pues se espera contar con la tecnología necesaria para su extracción en 2018 y poder comenzar a comercializar electricidad procedente de los hidratos de metano entre 2023 y 2027.

Uno de los métodos para realizar la extracción de los hidratos de metano es el de la despresurización, que consiste en inyectar dióxido de carbono (CO2) a fin de reemplazar el espacio que ocupa en el subsuelo. Japón realizó el primer experimento a nivel mundial de extracción de hidratos de metano aplicando este método en una zona marítima, del 12 a 18

\footnotetext{
${ }^{57}$ Plan Estratégico de Energía, p. 29
} 
marzo de 2013, llegando a extraer un total de 120.000 metros cúbicos, logrando un promedio diario de extracción de 20.000 metros cúbicos.

De ser posible la utilización de este método a gran escala, podrían cubrirse las tres "Es" del trilema energético japonés: la de la seguridad energética por contar con recursos propios, la de eficiencia económica al contar con una fuente energética autóctona y la de medio ambiente (environment) al contribuir a la reducción de gases de efecto invernadero.

\section{Transporte: ¿baterías de hidrógeno o de ion-litio?}

Junto con el sector industrial, uno de los sectores que más petróleo consume es el del transporte ${ }^{58}$, tanto el de mercancías como el de personas. El gobierno japonés ha apostado por incentivar la compra de vehículos menos contaminantes gravándolos con un tipo impositivo menor y fomentando mediante subsidios la compra de automóviles eléctricos para las flotas de taxis.

Con el lanzamiento al mercado de su modelo Prius en 1997, que es el más vendido en Japón, Toyota ha sido pionera en la fabricación de automóviles híbridos. En breve lanzará al mercado su modelo Mirai, un coche familiar bastante más económico que el Prius con el que espera ponerse a la altura del ix35 de la coreana Hyundai y cuya autonomía es mayor (350 km) un avance frente a su Prius, o al Leaf de Nissan cuya autonomía es de aproximadamente 150 kilómetros por carga. La autonomía sigue siendo la mayor preocupación tanto de usuarios como de fabricantes.

En un reciente artículo la BBC proclamaba abierta la batalla por establecer un estándar para la fabricación de vehículos de emisiones cero entre dos de los grandes fabricantes de automóviles japoneses: Toyota y Nissan. Mientras Toyota apostó en su día por las baterías de hidrógeno (fuel cells), Nissan ha optado por baterías de Ion-litio por contar con la ventaja comparativa de ser recargables con electricidad, por lo que no necesitarían de la construcción de una infraestructura de recarga específica, bastaría con enchufes más potentes.

\section{El debate sobre el mix energético post-Fukushima}

Antes del accidente, se esperaba que la energía nuclear desempeñase un papel aún más importante, pues se consideraba un vehículo para reducir las emisiones de CO2 dentro de la propuesta japonesa "Cool Earth 50" cuyo objetivo era reducir en un 50\%, en relación a los niveles del año 2000, las emisiones de CO2 para el año 2050, llegando al $90 \%$ en $2100^{59}$. En este escenario, la energía nuclear cubriría el $60 \%$ de las necesidades energéticas del país, mientras que el $40 \%$ restante lo completarían los combustibles fósiles (30\%) y energías renovables (10\%). En el momento de la propuesta, el año 2008, el 85\% de la energía procedía de los combustibles fósiles, el $10 \%$ de centrales nucleares y el $5 \%$ restante de energías renovables.

Vivoda considera que estamos en un periodo de transición energética, gradual y compleja. "El sistema global se encuentra en las fases tempranas de una transición de combustibles fósiles intensivos en carbono hacia una variedad de substitutos, lo cual conlleva riesgos ambientales, estratégicos y económicos." ${ }^{60}$ No obstante, las transiciones no se producen de forma instantánea, son procesos que pueden llegar a tardar décadas, ya de por sí

\footnotetext{
${ }^{58}$ Según datos de la Energy Information Agency estadounidense, en su informe de Japón de enero de 2015, el sector industrial consume el $29 \%$ del petróleo, mientras que el sector transporte consume el $41 \%$.

${ }^{59}$ En la página web del Ministerio de Asuntos Exteriores japonés puede encontrarse varios documentos relativos a la propuesta. http://www.mofa.go.jp/policy/environment/warm/coolearth50/,

${ }^{60}$ Vivoda, op. cit., p. 137
} 
la adopción de innovaciones tecnológicas se hace de forma gradual con etapas en las que conviven los dos modelos: el nuevo y el que va a ser desplazado. Una transición que requiere no solo la adopción de nuevas tecnologías, sino también el desarrollo de infraestructuras podría llevar décadas.

Vivoda señala también que cuanto más arraigado está el uso de un tipo de energía determinado, más tiempo tarda en llevarse a cabo la transición energética que podría prolongarse durante décadas. Si bien, cabe añadir que el contexto y la capacidad disruptiva de la nueva tecnología también entran en juego. Así el descubrimiento de una fuente energética superior puede dar lugar a una transición energética rápida, como sucediera a finales del siglo XIX con la substitución del aceite de ballena por el petróleo. Sin embargo, las crisis tan solo alteran el panorama de forma tangencial, señala Vivoda antes de concluir que el patrón histórico suele volver a los niveles anteriores a la crisis en cuestión de dos décadas, poniendo como ejemplo el movimiento pendular de Japón cuya demanda de petróleo había caído significativamente a mediados de la década de 1980, regresando a porcentajes pre-crisis a mediados de la década siguiente.

Las peculiaridades del sistema energético japonés unidas a las dificultades que conlleva toda transición energética ponen de manifiesto que la próxima transición energética no será rápida y que a medio plazo los combustibles fósiles seguirán siendo cruciales para todo gobierno que quiera garantizar su seguridad energética nacional. Tampoco se vislumbra por el momento en el horizonte energético japonés ninguna fuente energética que pueda desplazar a los combustibles fósiles.

A pesar de la actual parada en frío de la mayoría de los reactores nucleares, a juicio de la mayoría de los expertos japoneses en cuestiones energéticas, tanto dentro del gobierno y la administración pública, como en las empresas del sector y el mundo académico, la importancia de lo nuclear en el mix energético del país es incuestionable pues estaba alineada con los tres objetivos del triángulo energético: seguridad del suministro energético, metas climáticas/ambientales y competitividad económica del conjunto del sector energético ${ }^{61}$.

Con los escasos recursos energéticos propios con que cuenta Japón, en la actualidad tan sólo puede hacer frente a un $9 \%$ del consumo total de energía primaria del país, una cifra inferior a la de 2011. Antes del accidente nuclear de Fukushima, cuando la energía nuclear entraba en la ecuación energética, sus recursos propios satisfacían el $20 \%$ de la demanda ${ }^{62}$. A causa de la paulatina parada en frío de los reactores nucleares tras el desastre, el último de los cuales dejó de operar a comienzo de 2012, las empresas eléctricas reactivaron algunas de sus antiguas centrales convencionales y sustituyeron el uranio por el gas natural licuado (GNL) y los combustibles fósiles.

En el verano de 2011 se pidió a los consumidores que moderaran su consumo y asimismo, a fin de no sobrecargar la red, se procedió a realizar cortes programados, los precios de la electricidad subieron al tiempo que Japón aumentaba su dependencia de los combustibles fósiles, convirtiéndose en el tercer mayor importador neto y consumidor de

\footnotetext{
${ }^{61}$ Umbach, Frank: “The Energy Security of Japan after Fukushima 3/11”, en Paul Midford y Espen Moe (eds.), (2014): The Political Economy of Renewable Energy and Energy Security: Common Challenges and National Responses in Japan, China and Northern Europe, Palgrave Macmillan, p. 55.

${ }^{62}$ Datos relativos a 2013 del think tank estadounidense EIA, Energy Information Administration, citados en su página web y procedentes de "EIA International Energy Statistics" y "BP Statistical Review of World Energy, 2014", en http://www.eia.gov/beta/international/analysis.cfm?iso=JPN
} 
petróleo a nivel mundial, por detrás de Estados Unidos y China (EIA). Además, de perfilarse como mayor importador mundial de gas natural licuado y segundo mayor importador de carbón por detrás de China (EIA).

En su Tercer Plan de Energía, de junio de 2010 (un año antes de Fukushima y vigente en el momento de la catástrofe) el METI había resuelto aumentar la autosuficiencia energética al $70 \%$ en 2030 , para lo que la energía nuclear estaba llamada a desempeñar un papel protagonista en la aplicación del plan, que conllevaría la puesta en marcha de nuevos reactores. A principios de 2011, la energía nuclear representaba casi el $30 \%$ de la producción eléctrica total del país y había planes de que se convirtiese en $41 \%$ en 2017 y llegase al 50\% en 2030 .

El Cuarto Plan Estratégico de Energía de 2014, que hace una revisión del anterior, considera que la energía nuclear continúa siendo fundamental para garantizar un suministro energético estable y asequible, además de un vehículo para combatir el calentamiento global, si bien el empeño de que se utilice de forma segura es destacable. Así, antes de volver a ponerse en funcionamiento los reactores nucleares deben superar un minucioso examen, con requisitos fijados por la nueva Nuclear Regulation Authority (NRA), un ente regulador bajo los auspicios del Ministerio del Medio Ambiente que funciona de forma independiente.

Un panel formado por 25 expertos en calidad de asesores METI, denominado "Comité Asesor para Recursos Naturales y Energía" (Advisory Committee for Natural Resources and Energy) y nombrado por el Gabinete del Primer Ministro en junio de 2011, se reunió por primera vez en octubre de 2011 para debatir posibles escenarios energéticos con horizonte 2030. Un año más tarde, a finales de junio de 2012, el panel de expertos hacía públicas sus conclusiones: Planteaban tres combinaciones energéticas diferentes y sus respectivas emisiones de gases de efecto invernadero: El primer escenario plantea la desaparición de la energía nuclear $(0 \%)$ del mix energético, el segundo que represente el $15 \% \mathrm{y}$, el tercero, entre el $20 \%$ y el $25 \%$. 


\section{Gráfico 7. Tres opciones de marketing mix en debate}

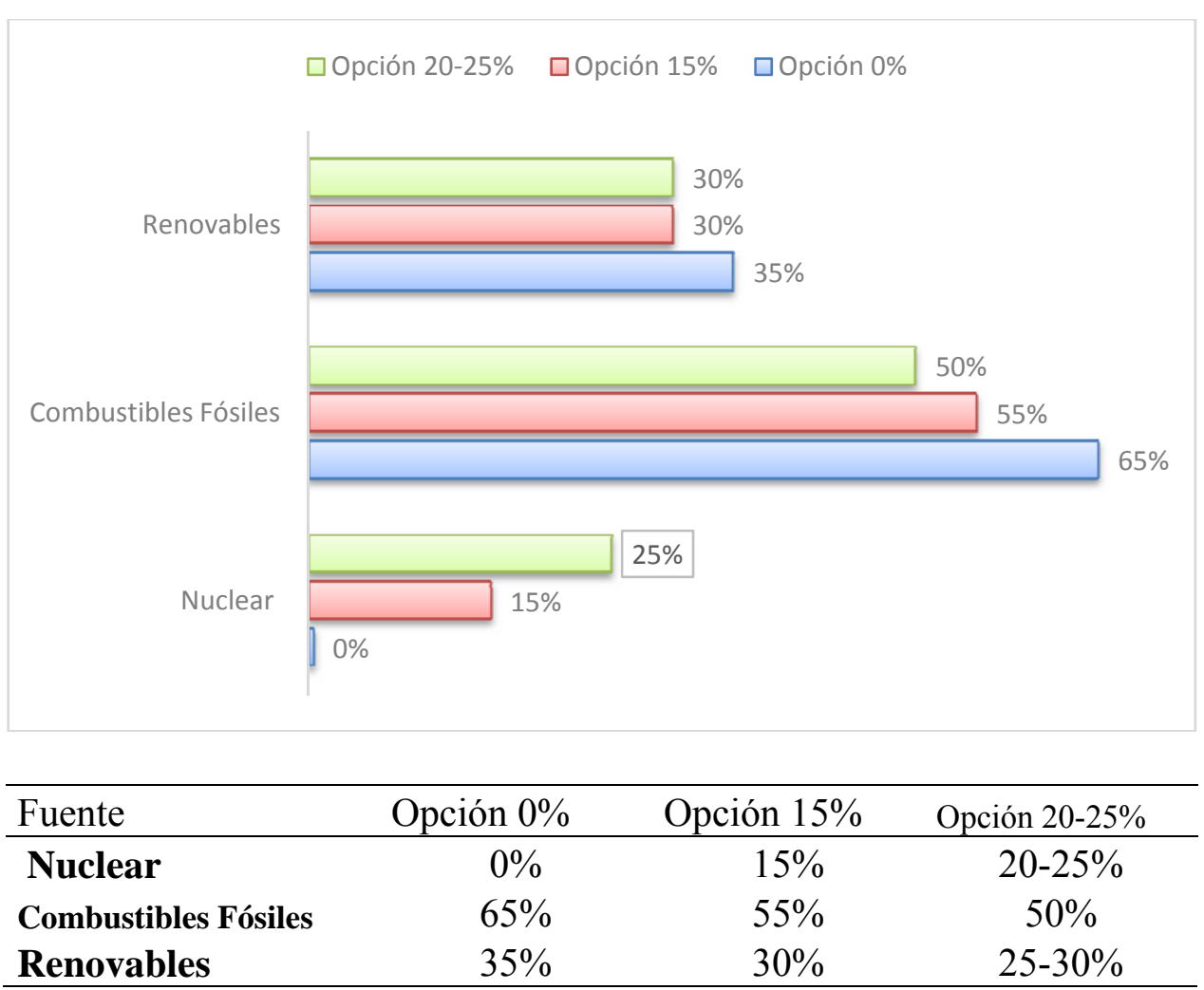

Fuente: Elaboración propia a partir de The Executive Committee of the Deliberative Poll on Energy and Environmental Policy Options (2012): Deliberative poll on energy and environmental policy options

Como se puede apreciar en la tabla y el gráfico que conforman la ilustración 9, en los tres posibles escenarios las renovables ocuparían un lugar destacado proporcionando casi una tercera parte de la energía primaria siendo su aportación mayor en caso de que se renunciara a la energía nuclear. Por otra parte, la aportación de los combustibles fósiles iría del 50 al 65\%, siendo también su aportación mayor en caso de renunciar a la energía nuclear y menor en caso de apostar por ella. Entre ambos, la opción intermedia del 15\% de energía nuclear conduciría a un uso más moderado de combustibles fósiles (55\%) y una apuesta por las renovables similar a la del escenario pro-nuclear.

Por una parte, cumplir los objetivos fijados en el marco del Protocolo de Kioto resulta imposible si no se recurre a la energía nuclear, de modo que quedaría descartado el primer escenario. Por la otra, según la revista Nature, Japón no planea construir ningún reactor en las próximas dos décadas por lo que para alcanzar el objetivo del $20-25 \%$ tendría que alargar la vida de los reactores existentes más allá de los 40 años (su vida útil), lo cual hallaría gran oposición tanto en la sociedad civil como en otros sectores. De forma que el escenario que de antemano parecía tener más posibilidades de ser el elegido era el del $15 \%$, un porcentaje que por otra parte podrían llegar a producir los reactores ya existentes si se respetase su vida útil ${ }^{63}$.

Por su parte, un grupo de ingenieros de la Universidad de Tokio y de la Agencia de Ciencia y Tecnología de Japón, analizaron las opciones publicadas por el Comité Asesor aplicando un modelo econométrico que ellos mismo habían desarrollado, basado en un modelo de demanda final de la energía y un equilibrio general computable, consideraban

\footnotetext{
${ }^{63}$ Cyranoski, David: “Japan considers nuclear-free future”, Nature, vol. 486 n7401 (6 de junio de 2012), p. 15.
} 
importante averiguar qué papel desempeñaban los patrones de consumo doméstico dentro del consumo total y llegaron a la conclusión de que la reducción de consumo en los hogares conllevaría una reducción de las emisiones de $\mathrm{CO} 2$, con independencia de las acciones asociadas a la energía nuclear. También señalaban la eficacia de otras medidas como las viviendas eficientes, los vehículos de bajo consumo o el marco legal establecido por el Programa "Top-Runner"64, una iniciativa que arrancó en el año 1998 con el objetivo de mejorar la eficiencia energética de los productos fabricados en Japón, desde electrodomésticos a automóviles, y que en 2009 se había convertido en uno de los pilares de la política climática japonesa ${ }^{65}$.

Vivoda concluye que la opción más factible para seguir siendo económicamente competitivo es la de volver a poner en marcha los reactores nucleares, si los gobernantes logran vencer la oposición de la opinión pública. A medio plazo, Japón depende de la importación de petróleo, carbón y GNL con visos de realizar una reducción gradual de la energía nuclear en el mix energético.

Gráfico 8. Mix energético: 2010-2014-2030

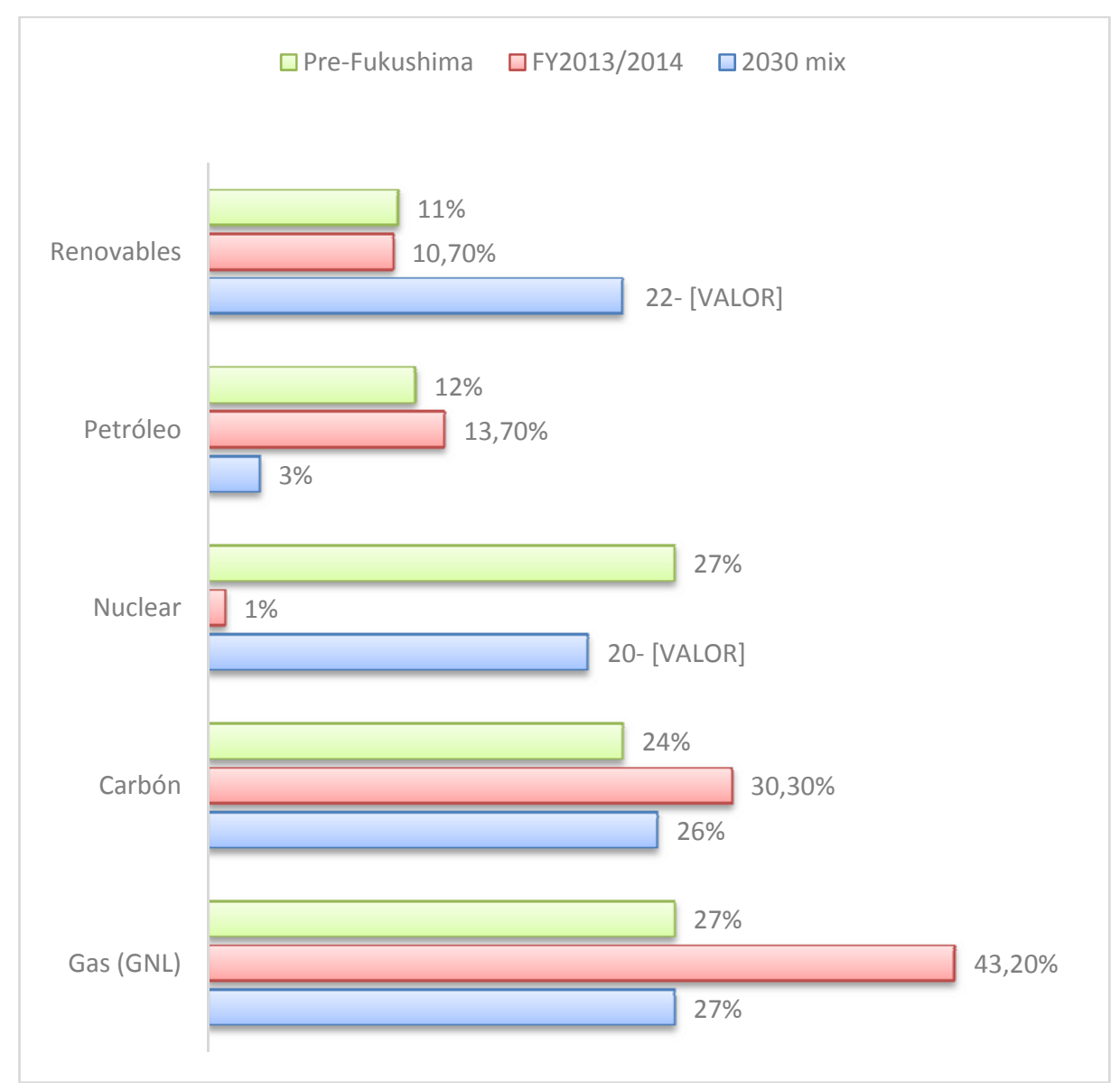

\footnotetext{
64 Matsuhashi, R., Takase, K., Yamada, K., y Komiyama, H.: "New energy policy and low-carbon society in Japan after the Great East Japan Earthquake", Chinese Journal of Population Resources and Environment, Vol. 11, $\mathrm{n}^{\circ} 1$ (marzo 2013), p. 68.

${ }^{65}$ Kimura, Osamu: “Japanese Top Runner Approach for energy efficiency standards”, SERC Discussion Paper No. SERC09035, CRIEPI (Central Research Institute of Electric Power), (2010), en http://criepi. denken.or.jp/jp/serc/discussion/index.html
} 


\begin{tabular}{lrrr}
\hline Gas (GNL) & $27 \%$ & $43,20 \%$ & $27 \%$ \\
Carbón & $26 \%$ & $30,30 \%$ & $24 \%$ \\
Nuclear & $20-22 \%$ & $1 \%$ & $27 \%$ \\
Petróleo & $3 \%$ & $13,70 \%$ & $12 \%$ \\
\hline Renovables & $22-24 \%$ & $10,70 \%$ & $11 \%$ \\
\hline Hidroeléctrica & $8,8-9,2 \%$ & & \\
Solar & $7 \%$ & & \\
Biomasa & $3,7-4,6 \%$ & & \\
Eólica & $1,70 \%$ & & \\
Geotérmica & $1-1,1 \%$ & & \\
\hline
\end{tabular}

A largo plazo, Japón apuesta por un desarrollo sostenido de las energías renovables, una apuesta que, según los críticos, podría lastrar la economía japonesa, pues supondría una pérdida de competitividad para las empresas japonesas con la consiguiente migración de empleos. De hecho, la administración Abe planea establecer un recorte de emisiones del 80\% para 2.050 con respecto a los niveles de emisiones actuales, introduciendo más capacidades de energías renovables y una mayor eficiencia energética.

\section{Conclusiones}

La continua preocupación de Japón por su seguridad energética a precios competitivos, a fin de satisfacer las necesidades de los distintos sectores económicos (industrial, transporte, comercial, hogares) es un buen ejemplo de lo que Calder denomina la "angustia energética" de Japón, un síndrome crónico fruto por una parte de su escasez de recursos propios y de la inestabilidad global, en parte consecuencia de la escalada de precios del petróleo (a pesar de su reciente bajada) y, por la otra, de crisis puntuales de suministro. Japón es el cuarto mayor consumidor de energía a nivel mundial y el lugar donde más caro resulta producirla, dado que debe importar la casi totalidad de recursos energéticos. Sin embargo, en el otro extremo, su eficiencia energética también se encuentra entre las mejores a nivel mundial.

La seguridad energética de este "gigante industrial de escasos recursos energéticos" se planifica minuciosamente a través planes estratégicos de energía que deben ser revisados al menos cada tres años. Son planes que elabora el METI y que aprueba el Gabinete, aunque la seguridad energética también está presente en la política exterior y de seguridad delineada por el Ministerio de Defensa en su Estrategia de Seguridad Nacional. En su búsqueda de la seguridad energética, Japón ha abogado por la diplomacia y la prevención de conflictos realizando una contribución pacífica a las relaciones internacionales y centrándose en sus metas económicas, aunque sin perder de vista el entorno cambiante y la necesidad de garantizar su seguridad nacional construyendo una arquitectura de seguridad integra apoyada sobre su alianza con Estados Unidos.

Resulta llamativo que aunque tanto el Cuarto Plan Estratégico de energía, como la Estrategia Nacional de Seguridad hablan de "seguridad" (Security) en su acepción de aseguramiento del suministro y de "seguridad" (Safety) en su dimensión de cumplir con estándares y normas que eviten accidentes, no mencionan la tercera "s" que es la de "salvaguardias" (Safeguards) o evitar un uso inadecuado (léase no pacífico, beligerante) de la

\footnotetext{
${ }^{66}$ Vivoda, op. cit.
} 
energía nuclear. La posibilidad de un ataque terrorista a una central nuclear o la substracción de material nuclear para la fabricación de bombas atómicas (las denominadas "bombas sucias"), son aspectos que entran dentro de esta tercera "s" y que tanto Japón como la comunidad internacional deberían contemplar al tratar seguridad tanto desde el punto de vista estratégico, como desde el punto de vista de la política energética.

El actual gobierno del PLD liderado por uno de los primeros ministros más carismáticos en lo que va de siglo XXI, Shinzo Abe, ha puesto en marcha una serie de reformas económicas a las que se conoce como Abenomics ${ }^{67}$ y cuyo objetivo es el de estimular la estancada economía japonesa (que entró en recesión en 2011) con medidas como el gasto en infraestructuras públicas, la reforma del mercado laboral o la inversión en empresas. Dentro de dichas iniciativas se encuentra también la reforma del sector energético y es que uno de los factores que contribuirá al éxito o el fracaso de tales medidas es el coste de la electricidad y, en consecuencia, el precio que se repercute sobre la industria, las empresas y las economías domésticas.

De ahí el interés que ha llevado a poner en marcha en agosto de 2015 el primer reactor nuclear, en la central nuclear de Sendai perteneciente a Kyushu Electric Company. Aunque la respuesta también habría que buscarla en la inclinación pro-nuclear del PLD y en sus vínculos con el poderoso lobby electronuclear, cultivados a lo largo de los años pues el PLD es el partido que ha llevado las riendas del país durante prácticamente toda la posguerra, con dos breves paréntesis a comienzos de la década de 1990, y de 2009 a 2012 en que ocupó el poder el PDJ.

La política energética japonesa ha puesto en marcha diversas medidas tendentes a promover una mayor conservación y eficiencia energéticas, así como a reducir su dependencia de las importaciones petrolíferas, que son las que mayor riesgo geopolítico presentan según hace constar en su último Plan Estratégico de Energía. Por su parte, la provincia de Fukushima está empeñada en dar un giro a su futuro y aspira a convertirse en un punto de referencia en materia de energías renovables, en las que ha invertido subsidios gubernamentales. En esta provincia se lleva a cabo un proyecto de investigación de energía eólica desde una gran plataforma flotante frente a la costa. Además, se han puesto en marcha dos centros de investigación (el Fukushima Renewable Energy Research Institute y el National Institute of Advanced Industrial Science and Tecnology).

El triple desastre parecía haber colocado a Japón en un momento catártico en el que debía decidir si su futuro será como su pasado, nuclear, o si bien las "3 E + S" de su trilema energético las marcarán las energías renovables. Cabía incluso cuestionarse si realmente nos encontramos ya ante La Cuarta Revolución o Revolución Verde, que sucederá a las tres primeras revoluciones históricas (agrícola, industrial y de las tecnologías de la información). Japón cuenta con cualidades técnicas, humanas y una visión de futuro capaz de poner en marcha una séptima ola de innovación y liderar la transición energética hacia energías renovables y alternativas. Por un lado, es uno de los países más tecnológicamente avanzados, y cuenta con valioso capital humano e intelectual, así como con universidades de élite en las que se forman e investigan ingenieros y científicos, y centros de investigación que durante décadas han situado al país a la vanguardia de la alta tecnología y la innovación. Por otro

\footnotetext{
${ }^{67}$ Las tres flechas con las que Abe ha puesto en marcha su política de reactivación económica son: (1) En materia de política monetaria, yen bajo a fin de incrementar la exportaciones, (2) estímulo fiscal, incrementando el gasto público y (3) reforma estructural de sectores clave como la agricultura, la salud y la energía, aumento de impuestos en dos etapas pasando el impuesto al consumo del 5\% al 8\% en 2014 y posteriormente al 10\%.
} 
lado, los japoneses se han embarcado en esta expedición con ese brío y con ese espíritu emprendedor que les caracterizan y que hicieron posible la "Revolución Meiji" a finales del siglo XIX y el "Milagro Japonés" a mediados del siglo XX.

Sin embargo, los últimos desarrollos apuntan a que en breve volverá a la senda nuclear. Tal y como se desprende de su apuesta por un mix energético en el que la energía nuclear suponga un $20-22 \%$ en 2030 y la puesta en marcha de los reactores que vayan superando las nuevas pruebas de resistencia tras la entrada en servicio del primerio en el verano de 2015. La energía nuclear ha sido uno de los pilares de la estrategia energética japonesa durante cuatro décadas y aunque el triple desastre podría haber supuesto un punto de inflexión, todo parece indicar que en un par de décadas Fukushima estará prácticamente desmantelado y la política energética japonesa habrá vuelto a su habitual "business as usual". No podemos pasar por alto que el futuro energético de Japón marcará de forma importante el futuro del planeta, dado que es un líder tecnológico cuyas innovaciones influyen no sólo en los países de su entorno (las economías de Asia, el continente en el que se encuentran potencias emergentes como China e India), sino también en el resto del mundo, tanto con el que comparte valores democráticos (Estados Unidos, Australia, la Unión Europea), como con la comunidad internacional en general. De ahí que habrá que seguir muy de cerca tanto sus decisiones en materia de política energética y de compromisos con el cuidado del medioambiente.

Las últimas propuestas de reducción de emisiones de gases de efecto invernadero han resultado bastante modestas tanto en comparación a las de otros países, como respecto a compromisos previos, debido a su actual dependencia de los combustibles fósiles a raíz del accidente nuclear de Fukushima y la parada en frío de los reactores. Japón, que es la tercera economía mundial y el quinto mayor emisor de gases de efecto invernadero, antes de la Cumbre del cambio climático de París de diciembre de 2015, la $21^{\text {a }}$ "Conference of Parties" o COP21, anunció su intención de realizar para 2030 reducciones del $26 \%$ en relación a niveles de 2013; mientras que Estados Unidos tenía intención de reducir entre 26 y $28 \%$ en relación a 2005 para 2025, y la Unión Europea se planteaba reducir $40 \%$ en relación a los niveles de 1990 o 35\% en relación a los de 2005 .

De la Cumbre debía salir un nuevo régimen que sustituyese al Protocolo de Kioto y, a pesar de las críticas, Japón hizo un esfuerzo por liderar los compromisos de reducción voluntaria de emisiones y llegar a acuerdos. No obstante, dada por una parte su dependencia de los combustibles fósiles a corto y medio plazo; y, por la otra, que su alta eficiencia energética hace que el coste de seguir incrementando la eficiencia energética sea exponencialmente mayor, en estos momentos una buena opción para contribuir al objetivo de frenar el calentamiento global es la de exportar tecnología a los países en desarrollo. 